\title{
DIPTÉRES TORRENTICOLES PEU CONNUS : IV. - LES ATHERICIDAE (ÉCOLOGIE ET BIOLOGIE) DU SUD DE LA FRANCE [Brachycera, Orthorrhapha]
}

\author{
par A. G. B. Thomas ${ }^{1}$.
}

Des prélèvements sur fonds de pierres et de graviers ont été effectués en 237 stations réparties sur 108 cours d'eau. Atherix ibis a été récolté jusqu'à $2200 \mathrm{~m}$ d'altitude, Ibisia marginata $1500 \mathrm{~m}$ et Atrichops crassipes $900 \mathrm{~m}$. Les principaux facteurs limitant la répartition des trois espèces sont :

1) la température de l'eau qui doit atteindre en été un minimum de 13$14^{\circ} \mathrm{C}$ pour $A$, ibis, de $11-12^{\circ} \mathrm{C}$ pour $I$. marginata, de $18-19^{\circ} \mathrm{C}$ pour A. crassipes ;

2) la pente (les ruisseaux sont plus rarement colonisés au-delà de $25 \%$ ) en corrélation directe avec la stabilité des pierres du fond entre lesquelles les larves d'A. ibis et d'I. marginata vivent étroitement insérées. Dans ce biotope précis, une taille réduite et une faible section transversale constituent une facteur favorable à l'installation ou au maintien des espèces dans les cours d'eau à forte pente (Hexatoma et Dicranota comparativement à A. ibis, divers Oligochètes comparativement à Eiseniella tetraedra). A. crassipes, essentiellement psammophile, vit en courant lent ou même temporairement nul ;

3) Ia présence de branches d'arbres à feuilles caduques au-dessus de l'eau, nécessaire à I. marginata pour accomplir son cycle de développement. Cette espèce pond sur la face inférieure des feuilles de plusieurs essences, en particulier de Corylus avellana. La tendance à une oviposition collective est nette, la même feuille pouvant porter jusqu'à plus de 400 pontes.

De l'amont vers l'aval on rencontre done : soit la succession ibis - marginata sous l'action prédominante de l'altitude (étages de végétation), soit, toujours en couvert de feuillus, la succession marginata-ibis par faible température estivale à altitude modérée.

La nymphose, toujours aérienne, a lieu le plus souvent dans les Bryophytes bordant le cours, à une distance de l'eau de 0,5 à $2 \mathrm{~m}$ chez $I$. marginata, dans les Bryophytes mais aussi dans les graviers émergés chez $A$. ibis, dans le sable chez $A$. crassipes. Elle dure de 10 à 12 jours à $19^{\circ} \mathrm{C}$ chez A. crassipes.

\section{Poorly known torrential Diptera : IV. - Athericidae of the south of France (ecology and biology) \\ (Brachycera, Orthorrapha).}

Samples from stony and gravel bottoms were taken at 237 stations distributed in 108 water bodies. Atherix ibis has been collected at altitudes up to $2200 \mathrm{~m}$, Ibisia marginata up to $1500 \mathrm{~m}$ and Atrichops crassipes up to $900 \mathrm{~m}$. The principal factors limiting the distribution of the three species are :

1) the water temperature which must reach a minimum in summer of $13-$ $14^{\circ} \mathrm{C}$ for $A$. ibis, $11-12^{\circ} \mathrm{C}$ for $I$. marginata, and $18-19^{\circ} \mathrm{C}$ for $A$. crassipes ;

1. Laboratoire d'Hydrobiologie, Université Paul-Sabatier, 118, route de Narbonne, 31077 Toulouse Cedex, France. 
2) the gradient (streams with a gradient greater than $25 \%$ are rarely colonised) in direct correlation with the stability of the substratum. Larvae of A. ibis and I. marginata live in narrow crevices between stones. In this particular biotope, a reduced size and low transverse cross-section are favourable factors for species living in streams with a steep gradient (Hexatoma and Dicranota compared with A. ibis, several oligochaetes compared with Eiseniella tetraedra). A. crassipes is essentially a psammophilic species that lives in slow-flowing or temporarily stagnant water;

3) the presence of branches of deciduous trees hanging over the water is necessary for I. marginata to complete its development cycle. This species oviposits on the lower surface of the leaves of several species, in particular Corylus avellana. A tendency towards colonial oviposition is evident with one leaf carrying up to $400 \mathrm{egg}$ masses.

From the upper to the lower reaches of rivers, there is either the succession ibis-marginata under the predominant influence of altitude (stages of vegetation), or, always with a covering of broad - leaved trees, the succession marginata-ibis at low summer temperatures at a reduced altitude.

Pupation always occurs out of water and occurs frequently in bryophytes at a distance of $0.5-2 \mathrm{~m}$ from the water in the case of $I$. marginata, in both bryophytes and exposed gravel in the case of $A$. ibis, and in sand in the case of A. crassipes. The pupal stage lasts $10-12$ days at $19^{\circ} \mathrm{C}$ in $A$. crassipes.

La biologie et surtout l'écologie des Athericidae - en particulier de leurs larves — n'ont été l'objet que d'un nombre de travaux très réduit à l'échelle mondiale et sont fort mal connues. Pratiquement aucune étude importante n'a été consacrée aux espèces paléarctiques, exception faite de la belle série des publications du Japonais Nagatomi (1958 à 1962). En Europe, hormis quelques citations dans des listes faunistiques, les contributions des hydrobiologistes à la connaissance de cette famille sont rares et presque toujours brèves. Mentionnons Pomeisl (1953 a et b), Osmera et Spitzer (1969), Vaňhara (1975) et surtout Neveu (sous presse).

La récolte de larves, nymphes, imagos et pontes en 237 stations de prélèvement réparties sur 108 cours d'eau permettent de préciser ici quelques aspects de la répartition et de la biologie des trois espèces du Sud de la France. La taxonomie et la détermination des adultes, des nymphes et des larves, puis le régime alimentaire de ces dernières ont été traités précédemment (Thomas $1974 \mathrm{a}$ et $\mathrm{b}, 1975 \mathrm{~b}$ ). Il a été souligné (Thomas 1974 a) combien peu satisfaisante était la combinaison Atherix marginata utilisée jusque-là. Quelques mois plus tard, la proposition de Stuckenberg (1974) d'adopter le nom générique Ibisia Rondani (1856) a apporté une solution souhaitable à ce problème de nomenclature et la combinaison Ibisia marginata est reprise ici.

Je remercie MM. : F. Vaillant (Grenoble) qui m'a communiqué du matéricl des Alpes, N. Giani (Toulouse) et M. Lafont (Besançon) qui ont respectivement déterminé les Oligochètes et les Hirudinées, H. Tachet (Lyon) pour ses photos d'Atrichops crassipes. 


\section{I. - ELEVAGES}

Pendant plusieurs mois, des élevages de larves en eau courante ont été nécessaires pour les études de taxonomie, du régime alimentaire et du comportement, en particulier des larves vis-à-vis du substrat. Des baes en plastique, à fond opaque ou transparent suivant les cas, ont été utilisés (photo 1). Pente variant de moins de 1 à $15 \%$, débit de 0,1 à $6 \mathrm{l} / \mathrm{mn}$. Deux grilles dans la partie aval du bac, et un couvercle amovible grillagé au-dessus. Fond plat ou en $\mathrm{V}$ ou en $\mathrm{U}$ plus ou moins accentués.

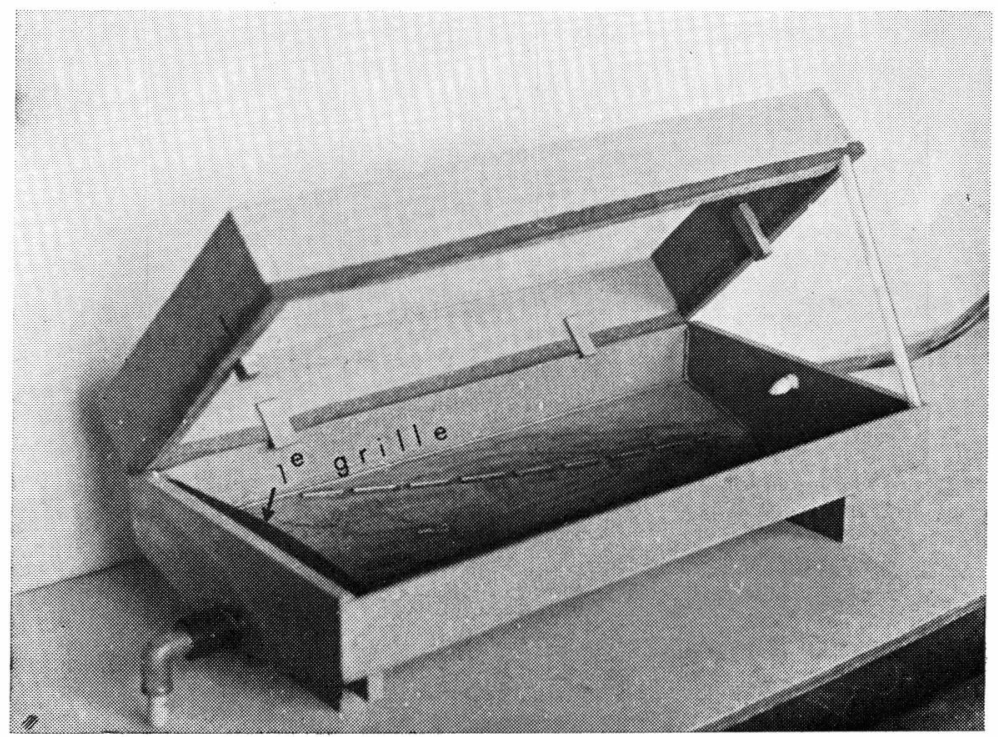

Рното 1. - Bac d'élevage en position ouverte.

\section{II. - STATIONS ETUDIẼES}

Stations et cours d'eau sont regroupés dans le tableau 1 où figurent aussi les principaux paramètres écologiques des Athericidae.

\section{Remarques :}

1. Pour une description plus complète de la plupart des stations, se reporter aux travaux suivants auxquels les lettres de la $4^{\mathrm{e}}$ colonne correspondent respectivement: AT à Thomas (1975 a), BL à Berthélemy et Laur (1975), CB à Berthélemy (1966), HD à Décamps (1967) et PL à Lavandier (1974). 
2. La pente moyenne indiquée concerne les environs de la station considérée, jusqu'à au moins une centaine de mètres en aval et autant en amont, chaque fois que cela a été possible.

3. Lorsque la station a fait l'objet d'un nombre de mesures de température important ou lorsqu'un thermomètre à maximaminima a été utilisé, la valeur retenue est soulignée : elle ne correspond pas à une température «record» mais à un maximum pratique obtenu pendant au moins deux à trois semaines. Les températures maximales relevées en l'absence de mesures estivales ne sont pas mentionnées.

4. Enneigement : une croix $(+)$ indique la présence d'une couverture de neige sur le cours d'eau lui-même pendant plusieurs semaines ou mois par an.

5. Végétation environnante (en particulier sur les deux premiers $\mathrm{m}$ des berges) : $\mathrm{P}=$ prairie, $\mathrm{C}=$ conifères, $\mathrm{F}=$ arbres à feuilles caduques $(\mathrm{PC}$ et $\mathrm{PF}=$ conifères et feuillus clairsemés ou rares en prairie).

6. La largeur moyenne des cours d'eau est mesurée en dehors des crues et des périodes d'étiage intense.

7. Les classes d'abondance utilisées par Décamps (1967) ont été reprises :

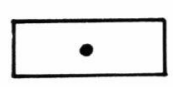

1 à 4

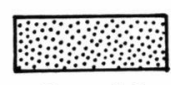

5 à 19

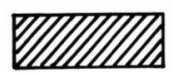

20 à 99

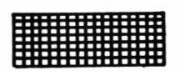

100 a 500

La lettre P signifie : présent mais rare.

Tous les prélèvements ont été faits sur fonds de pierres ou de graviers, au filet troubleau (vide de maille $0,2 \mathrm{~mm}$ ), mais une grande partie d'entre eux sans cadre de Surber : dans ce cas, les surfaces correspondantes ont été voisines de $0,5 \pm 0,1 \mathrm{~m}^{2}$. Les valeurs obtenues au filet de Surber ont été ramenées à $0,5 \mathrm{~m}^{2}$.

Lorsque plusieurs prélèvements ont été effectués à une même station, la classe retenue correspond à la valeur maximale obtenue, sauf toutefois si cette dernière s'écarte elle-même de plus d'une classe des autres valeurs. De plus, pour ne pas tenir compte des pullulations qui peuvent suivre les éclosions, les larves jeunes (moins de $4-5 \mathrm{~mm}$ ) ont été exclues des comptages.

8. Les données concernant les larves de Limoniidae des genres Hexatoma (2 sp.) et Dicranota ainsi que de Rhagionidae du genre Chrysopilus (2 sp.) ne sont pas quantitatives : seule la présence est mentionnée $(+)$. Habitat et forme du corps rendent sans doute 
en effet ces larves prédatrices directement concurrentes de celles des Athericidae dans les écosystèmes d'eau courante.

Matériel examiné : plus de 5000 larves.

- Répartition :

Atherix ibis : 3354 larves, 100 exuvies nymphales ;

Ibisia marginata: 1375 larves, 151 exuvies nymphales ; Atrichops crassipes : 188 larves, 11 exuvies nymphales.

- Régime alimentaire :

plus de 600 larves dont environ 500 A. ibis.

\section{III. - LA RÉPARTITION DES ATHERICIDAE : PRINCIPAUX PARAMẼTRES}

\section{1) Altitude maximale et végétation bordante.}

- Atherix ibis.

C'est, des trois espèces, celle qui remonte de loin le plus haut (tableaux 1 et 2) : elle est très abondante à la station 11 a $(2150 \mathrm{~m})$ et encore présente dans le même cours d'eau jusqu'à plus de $2200 \mathrm{~m}$. Les larves d'A. ibis peuvent se rencontrer en grand nombre, à la fois en prairie $(11$ a) et dans les bois de conifères $(38 \mathrm{a})$ ou de feuillus $(99)$. Des larves ont été récoltées jusqu'à $2350 \mathrm{~m}$ dans les Hautes-Alpes par M. Vaillant (Thomas 1974 a) et Bezzi a capturé des imagos à $2500 \mathrm{~m}$ dans les Alpes orientales (Séguy 1926).

\section{- Ibisia marginata.}

La limite altitudinale supérieure pratique de cette espèce se confond avec celle des arbres à feuilles caduques bordant les cours d'eau (tableau 1 : 40-40 a, 42-42 a, 50, 54, 56, 75-75 a ; tableau 2) s'il n'intervient pas de facteur limitant. Dans les Pyrénées, 1 . marginata n'excède donc que rarement $1500 \mathrm{~m}$ et par conséquent ne vit pratiquement jamais dans les cours d'eau recouverts par la neige l'hiver. Le tableau 2 fait apparaître une fréquence nettement supérieure au-dessous de $1000 \mathrm{~m}$ d'altitude, en particulier sous couvert de feuillus dense (F), ce qui s'explique par le mode d'oviposition (voir $\S$ IV). Brindle (1961) avait déjà remarqué la préférence de l'espèce pour les cours d'eau lents en régions boisées.

\section{- Atrichops crassipes.}

C'est une espèce de basse altitude : elle excède rarement $500 \mathrm{~m}$ (maximum relevé : $106 \mathrm{a}(930 \mathrm{~m})$ et $108 \mathrm{~b}(720 \mathrm{~m})$, sans captures de larves mais seulement d'imagos). La présence d'un couvert de 

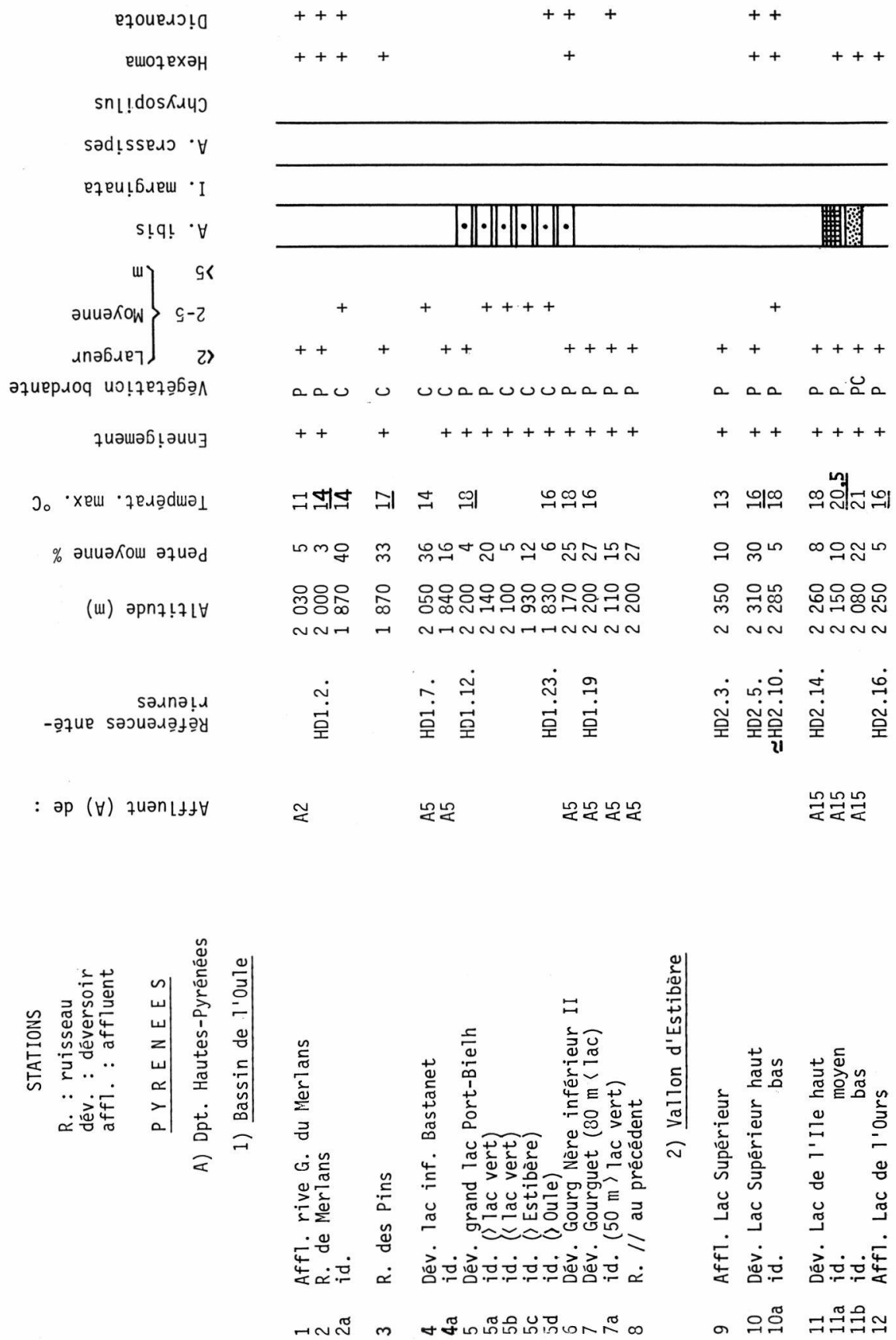

TABleaU I. - Répartition des Athericidae. Principaux paramètres des stations. 


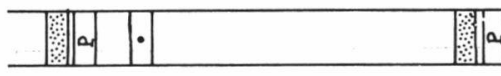

نصي

$++++$

ำ ำํำ

윰ำ굼ำ

옥융양융웡

n N N

ตํ่ล่ณ

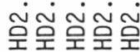

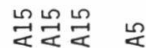

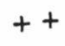

늠ㄴㅁㅇa a u

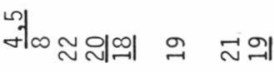

$\vec{v} \vec{v} \vec{v}$ 웅 $m \underset{\rightarrow}{m}$

육임의머앵 임 옹영 N NNA $N$ N-

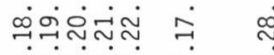

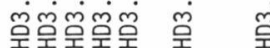

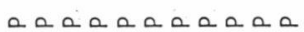

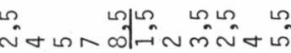

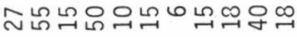

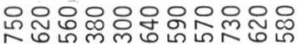

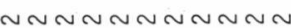

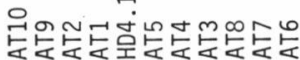

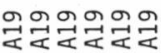

$a \cap \cup a \cap a$

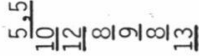

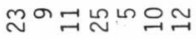

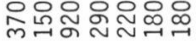
N $\sim \sim \sim \sim$

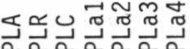

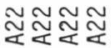

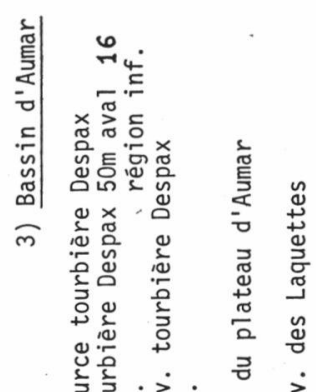

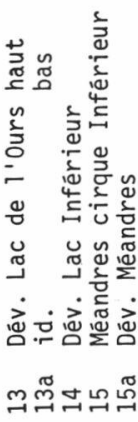

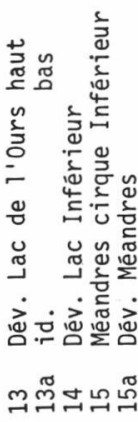

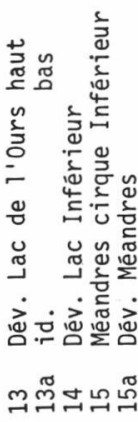

임

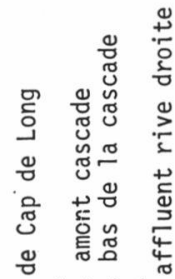

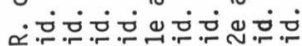
으응ํํㅇํํํํํํㅊำ

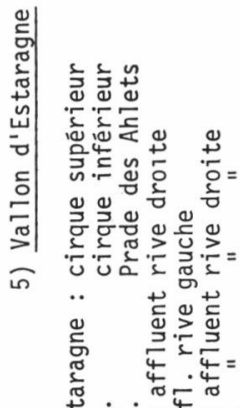

岃.ㅁㅜㅁㅀㅀ

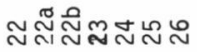




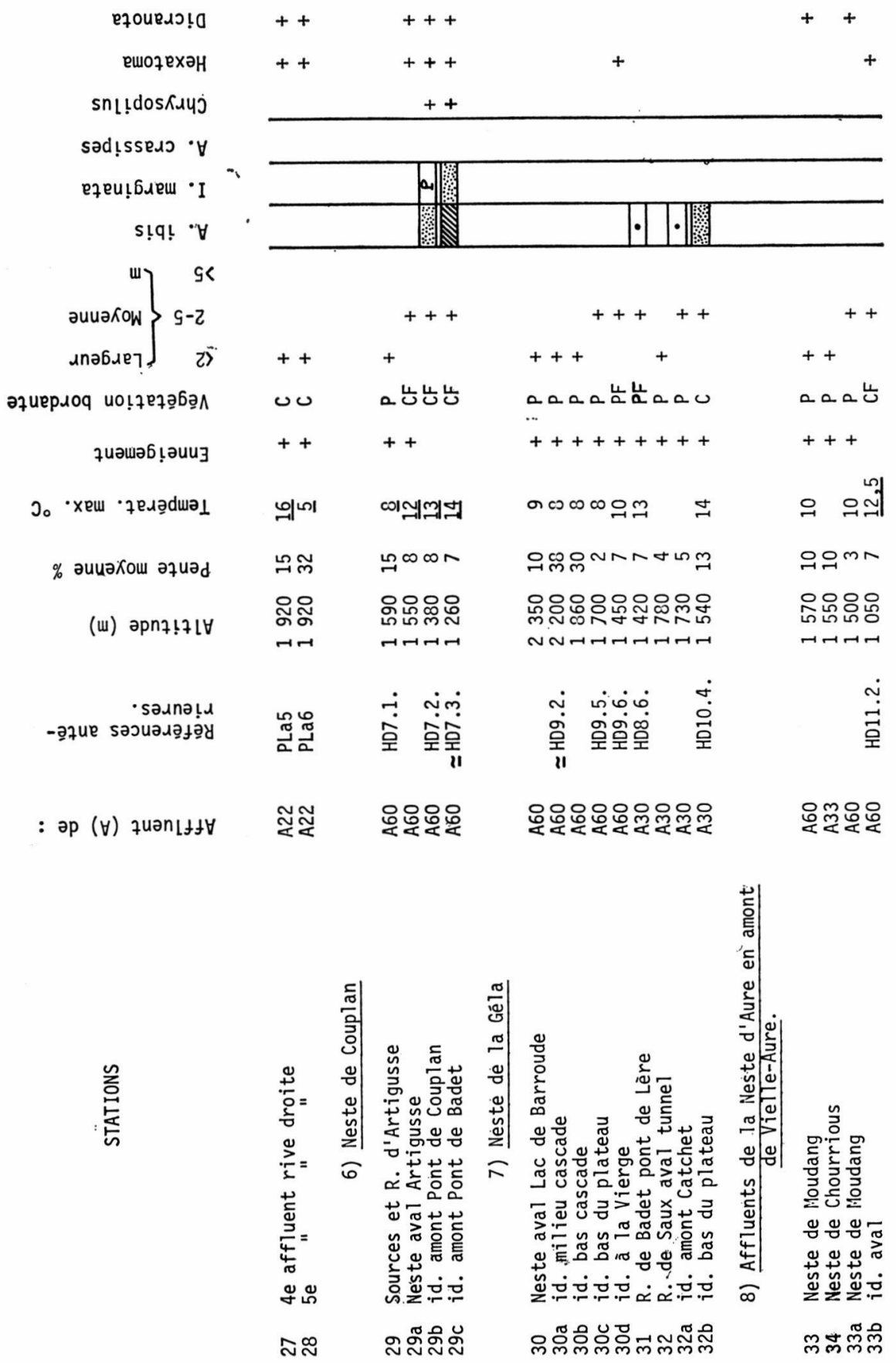




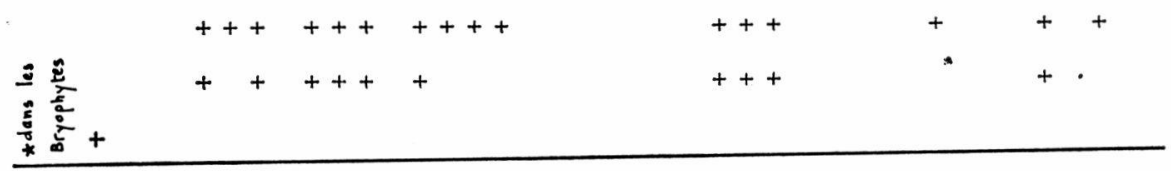

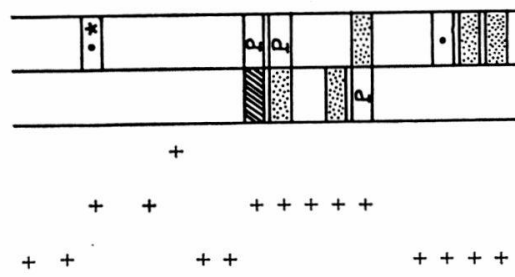

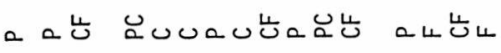

$+++++++++$

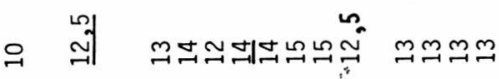

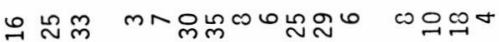

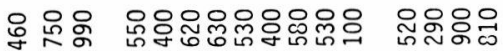
$\sim \rightarrow$

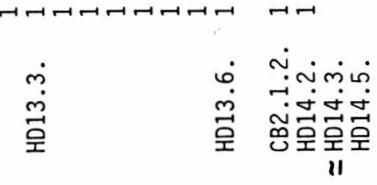

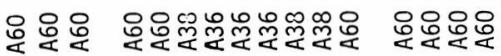
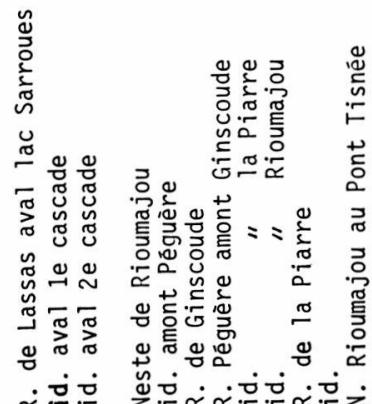

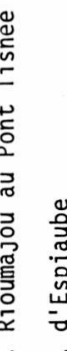

政

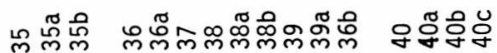

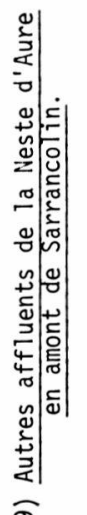

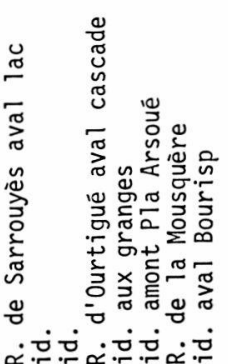

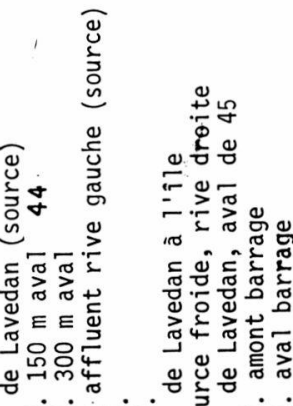

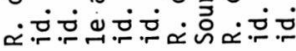

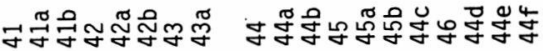




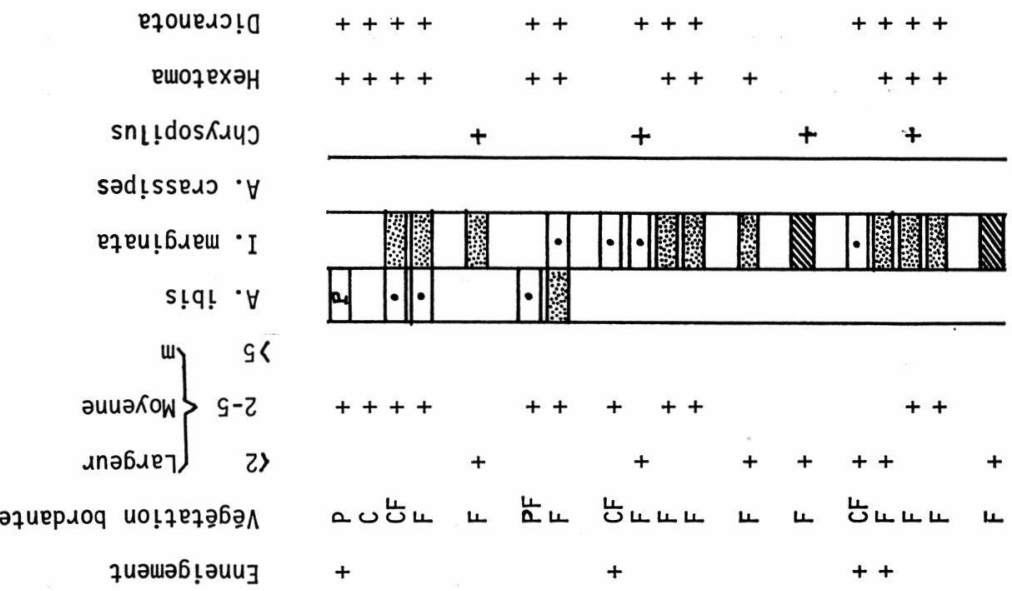

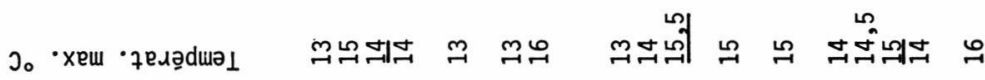

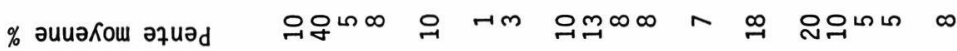

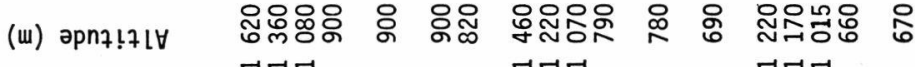
- saunata

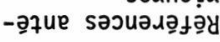
ن்.

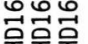

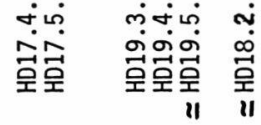
ஸ்ष्ठ்

: әр $(\forall)$ quәnLfft

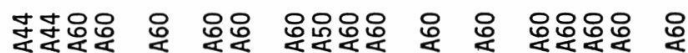

旁
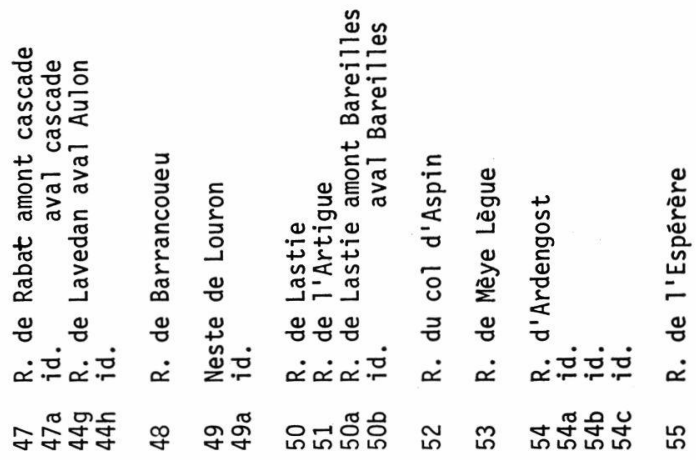

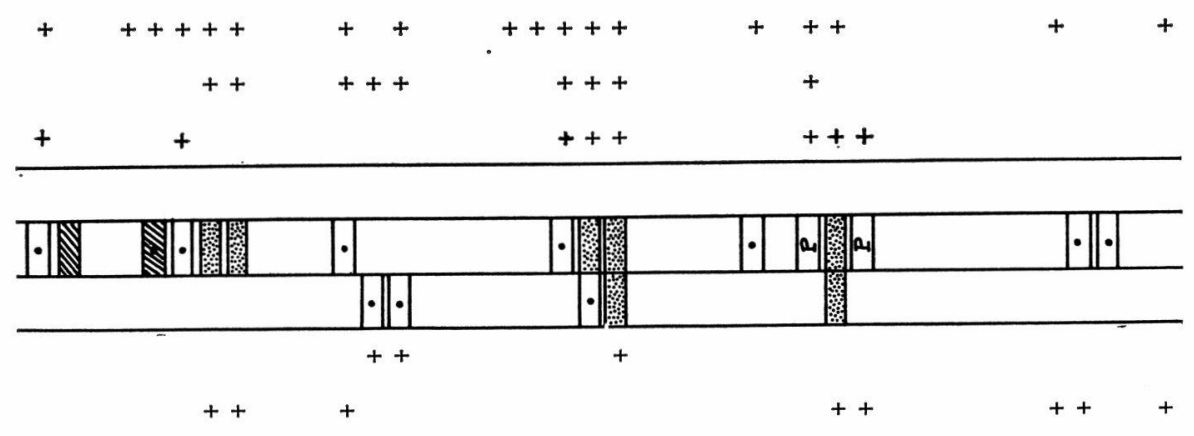

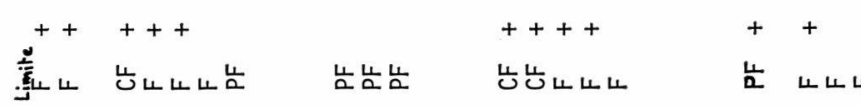

岕๘ル 。

\begin{tabular}{|c|c|c|c|}
\hline 뇩 뇸 & 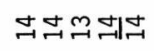 & 녹듀 & 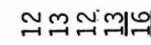 \\
\hline م & \&ீํำกナ & $m \beth \beth$ & 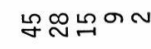 \\
\hline $\begin{array}{l}80 \\
8\end{array}$ & $\begin{array}{l}\text { 융앵양욧응 } \\
\text { r-1 }\end{array}$ & 옹융영 & 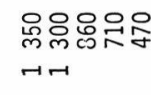 \\
\hline & 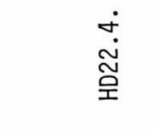 & 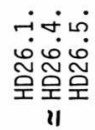 & \\
\hline
\end{tabular}

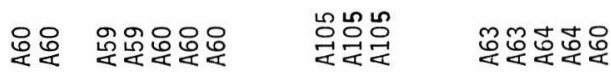

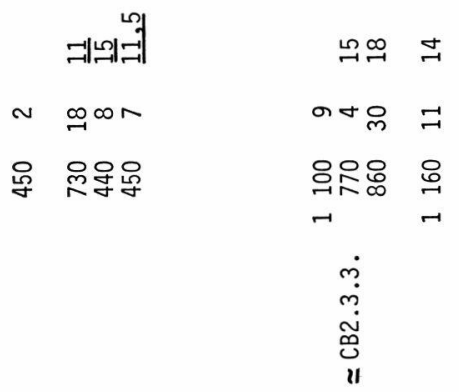

눈

운인원

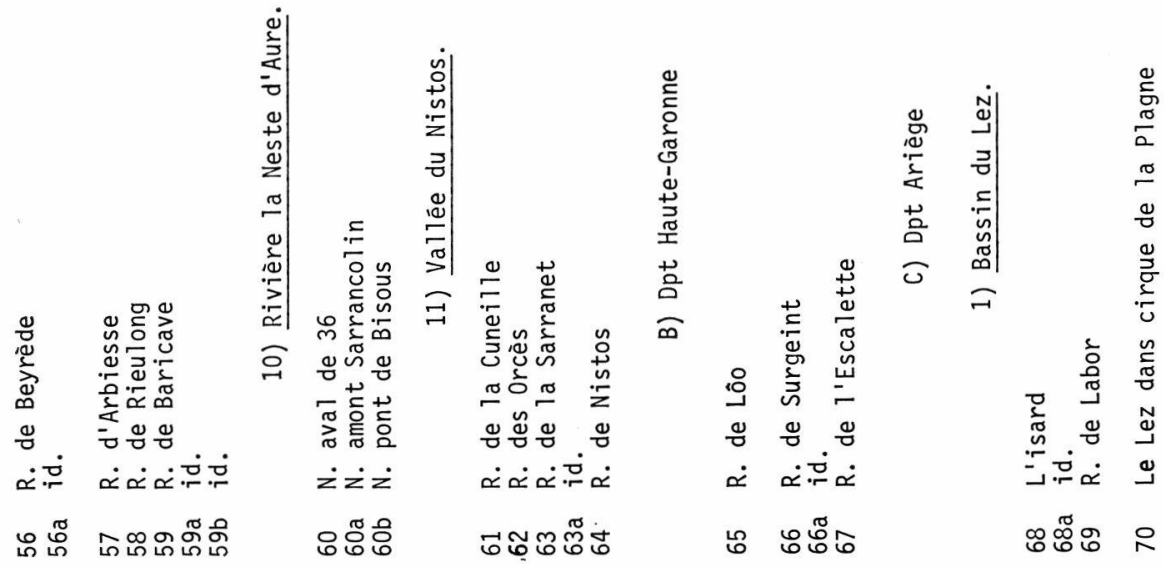




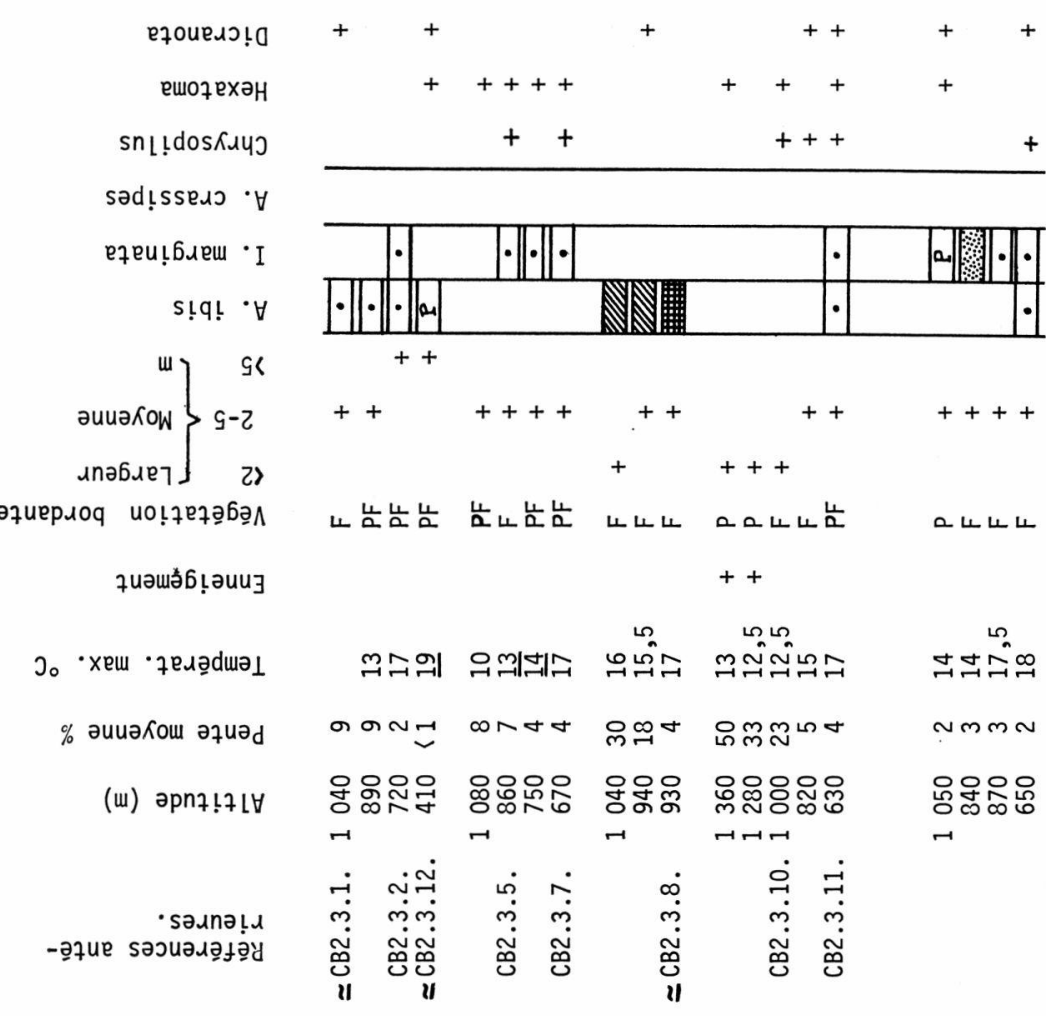

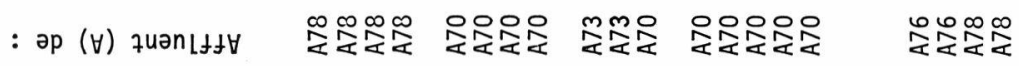

资

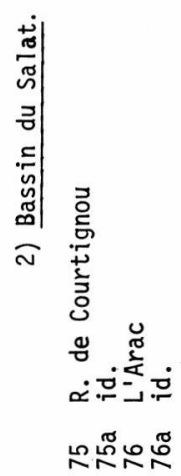



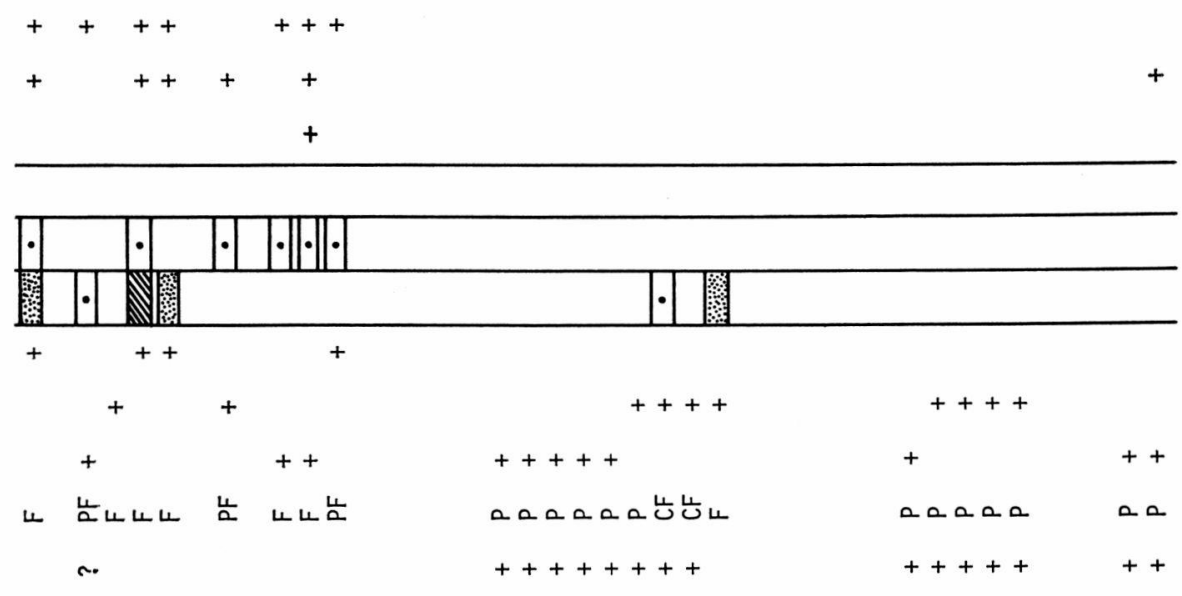

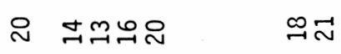 \\ $\because$ 마 0 NHЕ

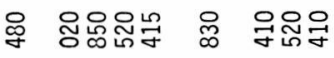 $\rightarrow$}

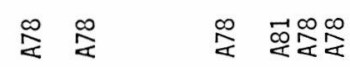

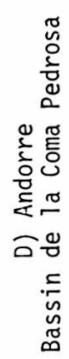

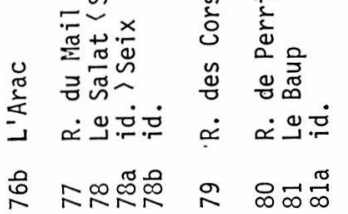

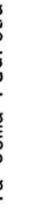

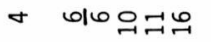

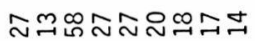

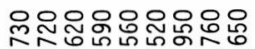

N $N$ N

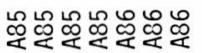

ชัญัช

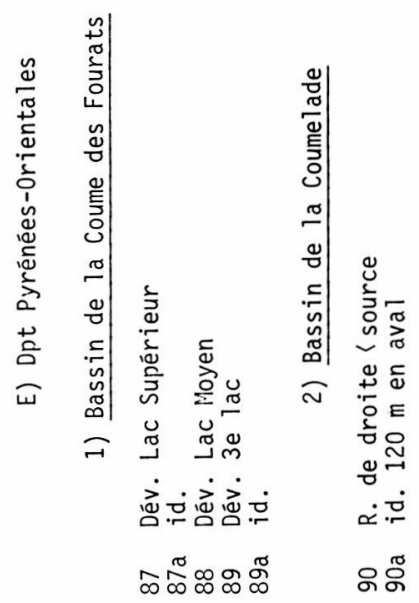




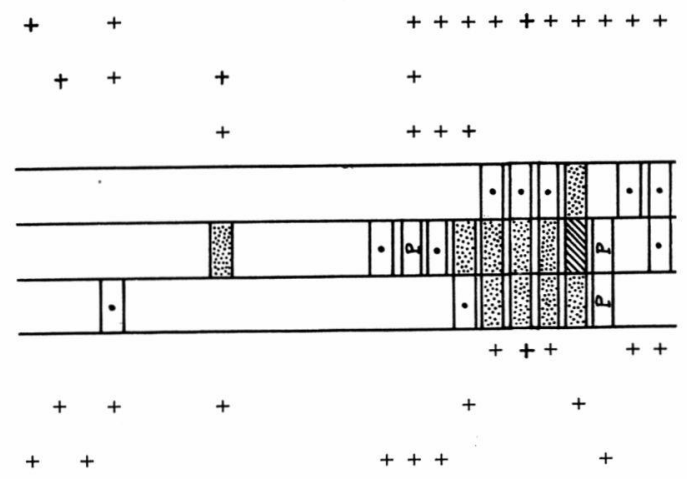

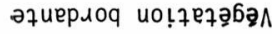

ロே岂ル

山

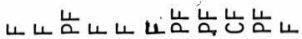

ұนวщә6เุนแ

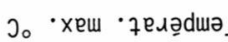

$ㅇ ㅡ$

さ

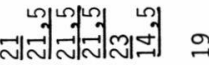

$\%$ әииәКош әұนә,

무ำ

$a$

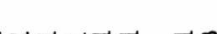

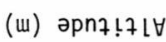

옹옴율ㄹ

잉

N

sวגกวเม

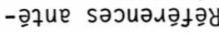

: әр ( $\forall)$ quәn Lft

ชัช ั

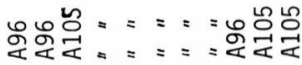

垈

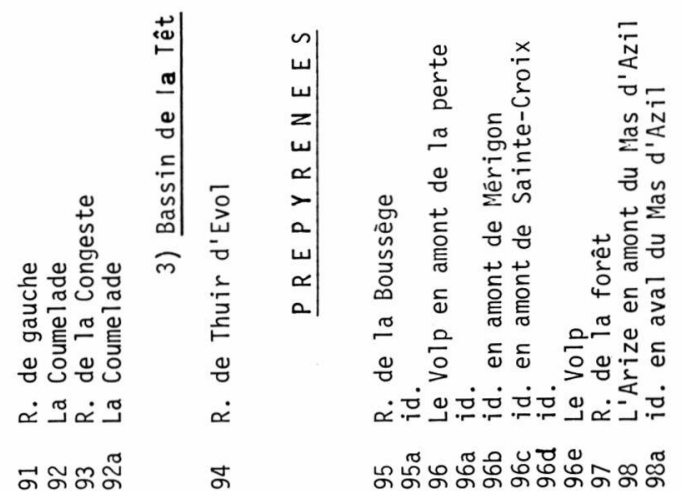




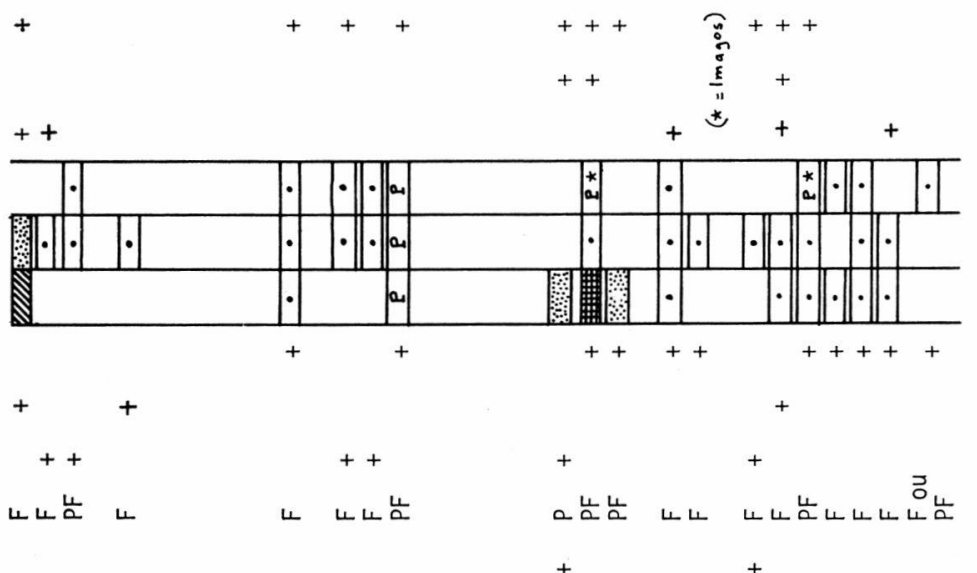

이드으 요

ㄴำㅉำ

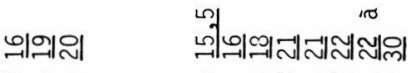

बrit in

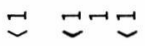

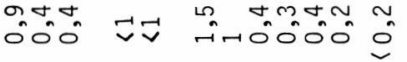

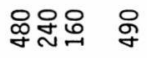

응 임영워

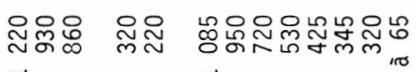

$\vec{\varpi} \vec{\omega} \quad \vec{\omega} \vec{\omega} \vec{\omega} \varpi \vec{\omega}$

弚

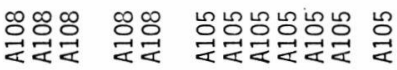

\begin{tabular}{l|l}
$w$ \\
$\alpha$ \\
0 \\
0 \\
$z$ \\
$w$ \\
$z$ \\
$\dot{b}$ \\
0 \\
$\vdots$ \\
$z$ \\
0 \\
$z$
\end{tabular}
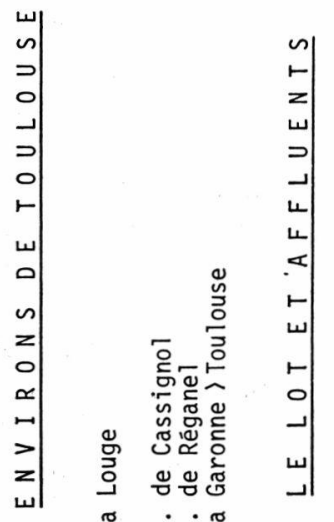

ญั้

훔

苟

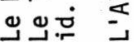

$\dot{\square} \dot{x}$

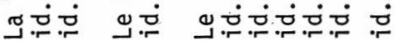

잉유 우

ฐ

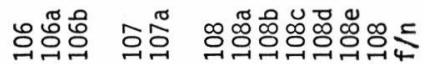

Tableau I (fin) 
feuillus dense ne se traduit pas par un accroissement de la fréquence (tableau 2).

\section{Conclusion.}

La succession des trois espèces en altitude décroissante est, dans l'absolu, A. ibis - I. marginata - A. crassipes (tableau 2). En réalité, dans un réseau hydrographique donné, elle est fréquemment modifiée par la température maximale atteinte par chaque cours d'eau.

D'autre part, dans les Pyrénées, les limites altitudinales atteintes par les espèces franchement torrenticoles des genres Hexatoma et Dicranota (Limoniidae) sont plus élevées que celles d'A. ibis (tableau 1 : 10-10 a, 22 a-28, 30 d, 33, $44 \mathrm{~b}$, etc.). En aucun cas les larves d'Athericidae ne font partie des quelques espèces qui composent les «peuplements-limites » à très haute altitude $(9,19$ à $22,30,35,44,45,82$ à 84,87 à 91$)$.

\section{2) Température maximale et largeur du cours.}

- Atheris ibis.

$A$ basse et moyenne altitude, cette espèce exige une température maximale pratique de l'eau - une valeur seuil en quelque sorte de 13 à $14{ }^{\circ} \mathrm{C}$ ( fig. 1 a ; tableau 1 : comparer 29 a et b, $32 \mathrm{~b}, 33 \mathrm{~b}$, $38 \mathrm{a}, 44 \mathrm{f}, 49,70 \mathrm{~b}, 97$ ). Cette température doit pouvoir être atteinte les jours de beau temps, au moins au cours des deux ou trois semaines les plus chaudes de l'été.

A haute altitude, ce seuil est beaucoup plus élevé, de l'ordre de 18 à $20^{\circ} \mathrm{C}$ (comparer 6,10 a, 11-11 a, 12-13 a, 17, 27). En conséquence, $A$. ibis ne peut coloniser que les cours d'eau exposés à une forte insolation l'été. Les amplitudes thermiques journalières estivales y sont toujours importantes à cause des basses températures nocturnes. En revanche, l'enneigement annuel peut être considérable (plus de 6 mois à 11 a). Mais cette espèce ne se rencontre pas dans les cours d'eau qui se réchauffent peu l'été : par exemple, elle est absente dans l'Estaragne et ses affluents (22 à 28). Un nombre annuel de l'ordre de 1300 degrés jours (27: Lavandier 1974, p. 211) paraît insuffisant pour son développement. Ce nombre peut être comparé aux 600 degrés jours annuels de la station 22 a où vivent Hexatoma bicolor et au moins une espèce du genre Dicranota. Dans les ruisseaux froids, l'installation de ces Limoniidae précède celle des Athericidae (tableau 1: 29 a, $40,42,44$ b $68,70,71,75,90-91-92)$.

Enfin, quelle que soit l'altitude, $A$. ibis se rencontre le plus souvent (plus de $75 \%$ des cas) dans des cours d'eau d'une largeur supérieure à $2 \mathrm{~m}$ (fig. $1 \mathrm{~d})$. 


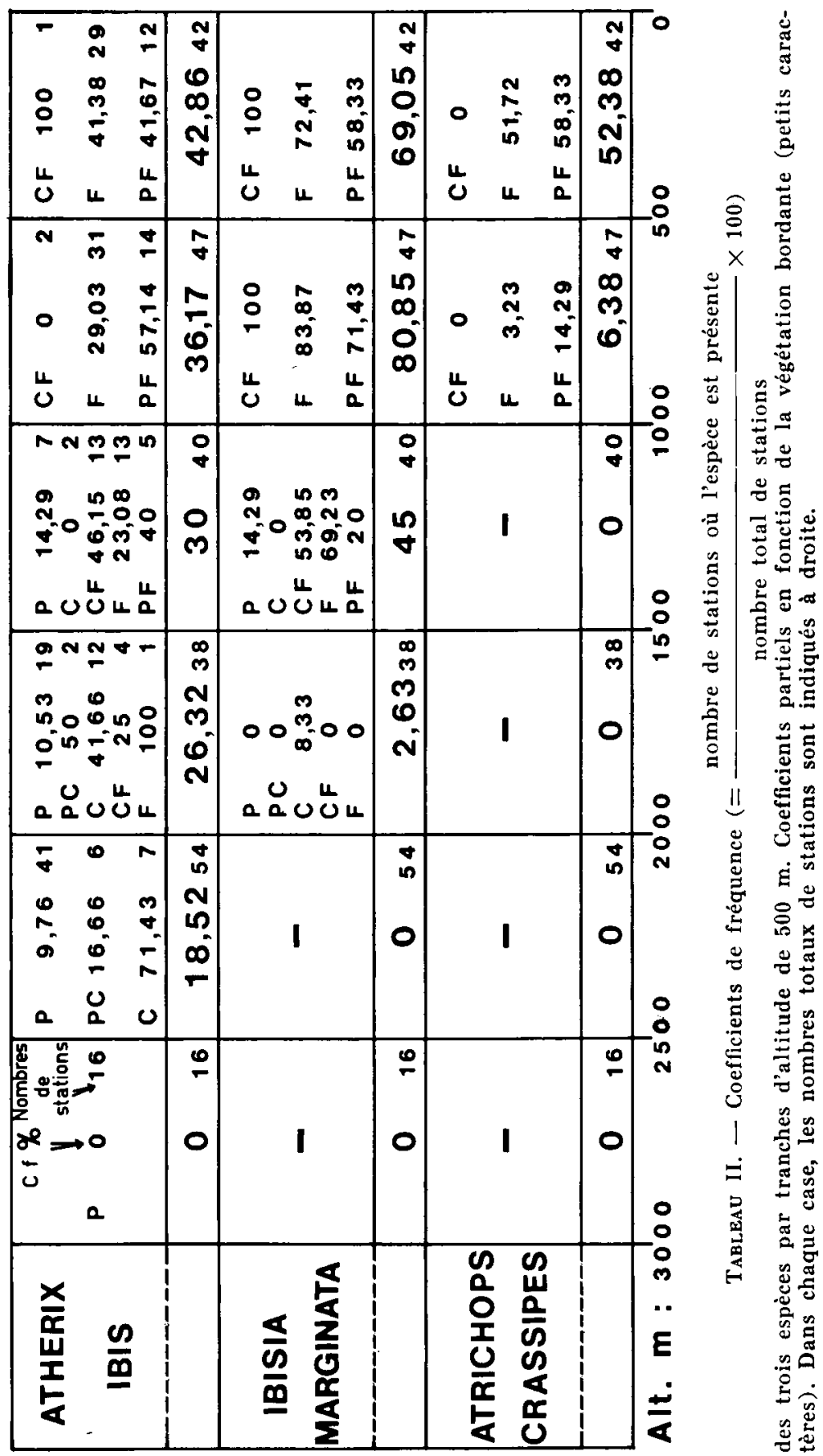



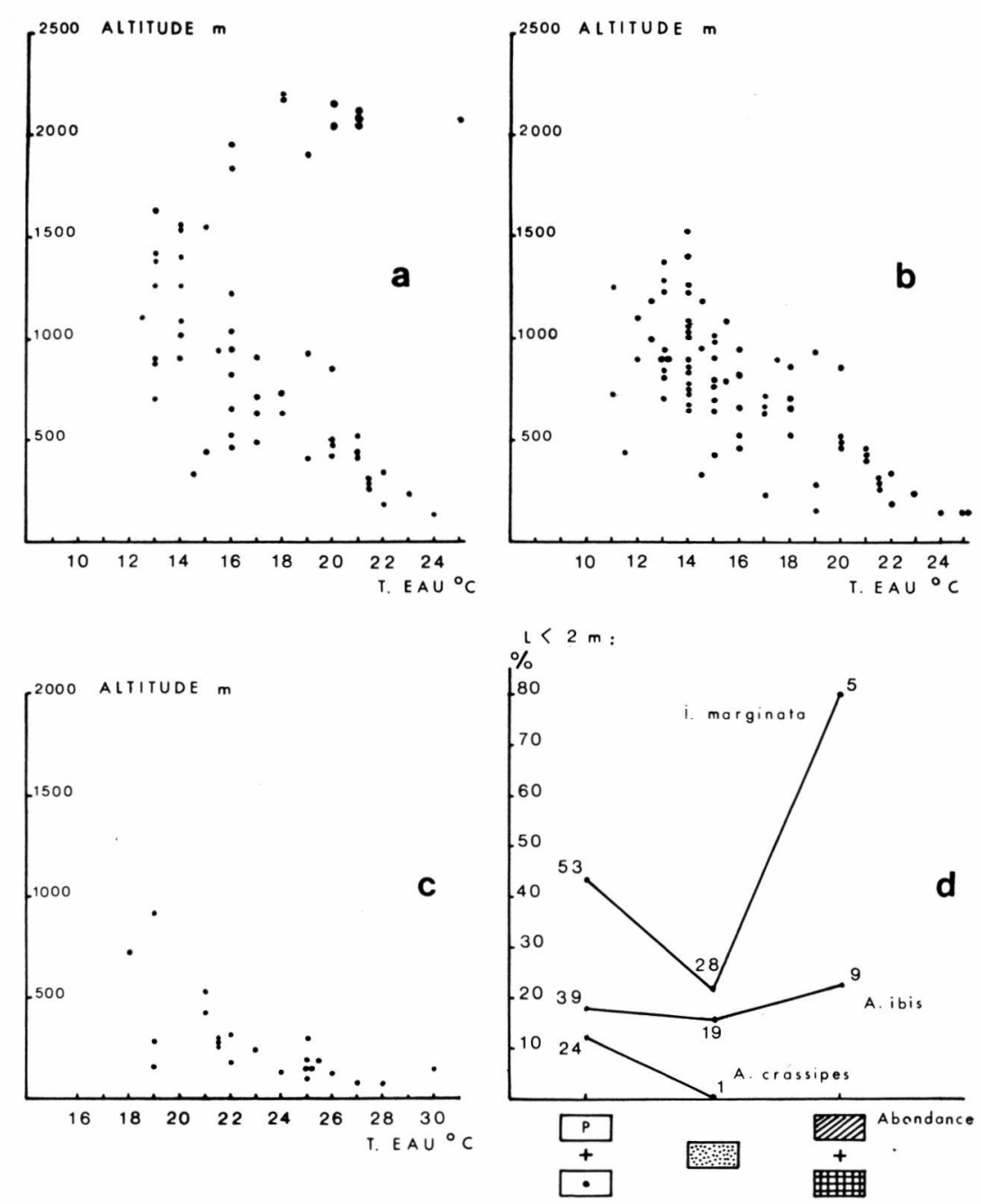

Fig. 1. - Températures maximales de l'eau aux stations colonisées par: $A$. ibis (a), I. marginata (b) et A. crassipes (c). d : pourcentages de stations de largeur de cours inférieure à $2 \mathrm{~m}$ en fonction des classes d'abondance (milieux : pierres ou graviers, à l'exclusion des sables proprement dits) ; nombres de stations indiqués.

\section{- Ibisia marginata.}

Malgré une limite altitudinale nettement inférieure à celle $\mathrm{d}^{\prime} \boldsymbol{A}$. ibis, les exigences de cette espèce vis-à-vis de la température maximale nécessaire sont moindres, avec un seuil de 11 à $12{ }^{\circ} \mathrm{C}$ (fig. $1 \mathrm{~b}$; tableau 1 : comparer $35 \mathrm{~b}, 42-42 \mathrm{a}-42 \mathrm{~b}, 63-63 \mathrm{a}, 66,67$ ).

I. marginata prolifère volontiers dans des ruisseaux étroits (fig. $1 \mathrm{~d}$; tableau 1 ), le plus souvent très ombragés et aux berges 
plus ou moins abondamment couvertes de Bryophytes à la limite de battement de l'eau. Ce dernier point est important pour la nymphose (voir $\S \mathrm{V}$ ).

\section{- Atrichops crassipes.}

Cette espèce vit dans des caux chaudes. Elle n'a été récoltée que dans des stations où la température maximale pratique de l'eau est comprise entre $18-19{ }^{\circ} \mathrm{C}$ au minimum el $30-31{ }^{\circ} \mathrm{C}$ (tableau 1 : 100-100 a, 106-106 a, $108 \mathrm{a}, \mathrm{b}, \mathrm{c})$. On la rencontre surtout dans des cours d'eau lents, souvent larges (fig. $1 \mathrm{~d}$ ) et par conséquent plus ou moins détériorés par les influences humaines.

\section{Conclusion.}

La succession marginata-ibis est donc rencontrée de l'amont vers l'aval lorsque les torrents les plus élevés sont trop froids en prairie ou sous les conifères — pour héberger $A$. ibis. Si la pente est favorable, la colonisation du réseau hydrographique commence alors dans les petits ruisseaux affluents froids, sous les arbres à feuilles caduques, par $I$. marginata dès que la température maximale de l'eau atteint 11 à $12^{\circ} \mathrm{C}$. A. ibis s'installe ensuite en général lorsque le lit s'élargit (cette espèce colonise en particulier le cours principal) et quand la température maximale de l'eau s'est accrue de 3 ou $4{ }^{\circ} \mathrm{C}(60-60$ a-60 b, 63-63 a-64, 75-76, 108-108 a).

En Autriche, dans la Mauerbach, Pomeisl (1953 a) a trouvé I. marginata plus en amont qu' $A$. ibis et, d'autre part, seule la première espèce était représentée dans quelques-uns des affluents. Dans une rivière du Japon, Nagatomi (1962) ne relève la présence d'A. ibis japonica que dans le cours principal alors que deux espèces du genre Suragina (voisin d'Ibisia) colonisent également les affluents et les fossés d'irrigation.

Les citations de larves d'Athericidac dans diverses listes faunistiques européennes accompagnées de relevés de températures estivales vont toujours de pair, à ma connaissance, avec des valeurs supérieures aux seuils donnés plus haut :

- A. ibis : Nietzke (1938), Pomeisl (1953 a), Zácwilichowska (1970), Neveu (sous presse);

- I. marginata : Pomeisl (t. c.), Dittmar (1955), Sowa (1965), Neveu (t. c.), Van̆hara (1975);

-A. crassipes : Neveu (t. c.) ;

- Atherix sp.: Jones (1949), Badcok (1949). 


\section{3) Pente.}

Le rôle de la pente est primordial. Il peut être mis en évidence par la comparaison des peuplements en $A$. ibis de deux ruisseaux coulant parallèlement, à la même altitude, à $200 \mathrm{~m}$ l'un de l'autre, avec des caractéristiques thermiques et chimiques assez voisines (11 a-11 b et 13-13a). Dans le premier ruisseau, la densité des larves décroît fortement vers l'aval avec une augmentation sensible de la pente; dans le second, les larves ne sont présentes que lorsque la forte pente initiale a été considérablement réduite.

Les Atherix ne disposent pas d'organes d'accrochage d'une grande efficacité et sont tributaires à un haut degré de la nature du substrat pour lutter contre le courant. Aussi, la stabilité des pierres du fond entre lesquelles - et surtout sous lesquelles $\boldsymbol{A}$. ibis et $\boldsymbol{I}$. marginata s'abritent, paraît-elle être un facteur déterminant de leur répartition après la température. Ces deux espèces deviennent beaucoup moins fréquentes sur fonds de pierres, à partir d'une pente de l'ordre de $20 \%$ (tableau 1). Les pentes supérieures sont presque toujours désertées par les Athericidae, probablement en raison de vibrations ou de déplacements insensibles du substrat qui doivent écraser les larves. En effet, l'observation de celles-ci en ruisseau artificiel à fond transparent révèle qu'elles s'insinuent en général profondément entre les pierres en épousant la forme des interstices.

La photo 2 montre une larve d'A. ibis à l'affût. Même dans ce cas qui exige des mouvements rapides, seuls la tête et le thorax demeurent réellement libres et très mobiles pour la détente. Le maintien de la larve contre le courant se fait par un aplatissement mécanique du corps entre les pierres du fond ou entre une pierre et la roche en place (dans le cas du ruisseau artificiel, entre une pierre et la plaque transparente $d u$ fond). Cet aplatissement des tergites abdominaux souples est visible sur la photo, alors que la région antérieure, elle, n'est pas aplatie et demeure de section à peu près circulaire.

Cet ancrage dans le substratum est efficace mais nécessaire car immédiatement après perforation, la proie est tirée à l'abri le plus loin possible sous la pierre. Elle doit être retenue pendant la durée de la succion qui peut excéder une heure (Thomas 1975 b).

Mais en contrepartie, les larves d'Athericidae, littéralement encastrées sur le fond, supportent sans doute très mal tout déplacement des pierres ou tassement du substratum (crues, fortes pentes). J'ai relevé, à plusieurs reprises dans les élevages, le cas de larves isolées qui, ne pouvant plus se dégager, étaient mortes, immobilisées par les pierres sous lesquelles elles s'abritaient. La 

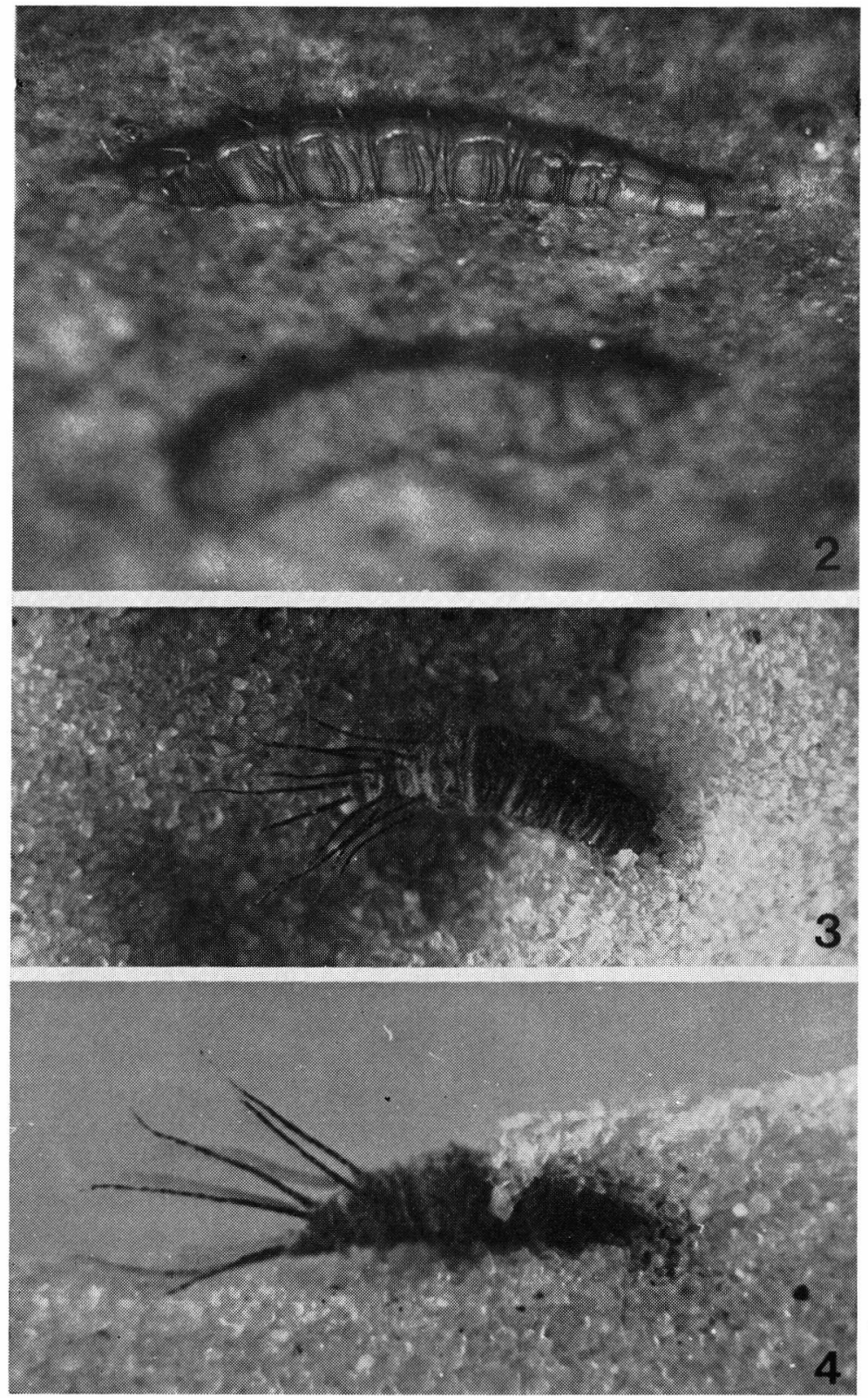

Рното 2. - Spécimen d'A. ibis à l'affût sous une pierre (photo prise par en dessous avec un fond transparent contre lequel on remarque l'aplatissement des tergites abdominaux).

Рнотоs 3 et 4. - Enfoncement d'une larve psammophile fouisseuse d'A. crassipes déposée sur un fond de gros sable (photos H. Tachet). 
force de ces larves et leur obstination sont considérables : sur substrat horizontal rigide (bacs d'élevage) mouillé mais hors de l'eau, des pierres d'une centaine de g, sous lesquelles elles cherchent à s'abriter, peuvent être ainsi très légèrement mais distinctement déplacées!

Pour les deux espèces $A$. ibis et $I$. marginata, la moyenne des 5 pentes les plus élevées du tableau 1 est très voisine de $25 \%$, ce qui paraît correspondre à un maximum pratique. Le maximum absolu, inférieur à celui de beaucoup d'insectes torrenticoles, ne semble guère dépasser $30 \%(18 \mathrm{a}, 35 \mathrm{~b}, 69,72)$. Les limites sont probablement inférieures avec des pierres de nature schisteuse plus glissantes les unes contre les autres, plus aplaties et surtout dépourvues de surfaces concaves - qu'avec des pierres de nature granitique. Les fortes pentes très stables (roche en place, grosses picrres) avec un faible débit toute l'année, autorisant un grand développement des Bryophytes aquatiques sur la totalité du lit, ne constituent qu'un cas particulier, plutôt rare (97).

\section{Remarque.}

Pour les espèces ne disposant pas ou guère d'organes d'accrochage efficaces (en particulier les Diptères Limoniidae et les Oligochètes), une faible section transversale du corps et, dans l'ensemble, des dimensions inférieures, constituent des éléments favorables au maintien des individus sur fonds de pierres par fortes pentes.

- Moyenne des 5 valeurs de pente les plus élevées du tableau 1 : pour Hexatoma bicolor $=39 \%$, pour Dicranota $s p p=41 \%$.

- Cas de fortes pentes où les Athericidae sont supplantés par les Limoniidae (avec des températures supérieures aux seuils donnés) : $57,61,62,74$.

\section{Exemple 1.}

Le tableau 3 montre la régression puis la disparition rapide d'A. ibis dans un affluent à forte pente d'un cours d'eau lui-même fortement peuplé en Athericidae. Le bilan thermique (température maximale, durée de l'enneigement) est voisin aux trois stations retenues. Dans l'affluent (st. 39 a et 39), il est intéressant de noter l'expansion concomitante d'une part des Hexatoma et Dicranota (Limoniidae), d'autre part des Rhyacophila et Plectrocnemia (Trichoptères), ce dernier ordre disposant de fortes griffes de fixation. A. ibis subsiste dans l'affluent à $50 \mathrm{~m}$ en amont du confluent mais en août la taille moyenne des larves y est très supéricure à celle des larves du cours principal et il est douteux que le cycle de développement soit bouclé à cette station. 


\begin{tabular}{|c|c|c|c|c|c|c|c|c|c|}
\hline 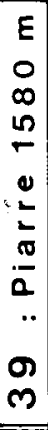 & 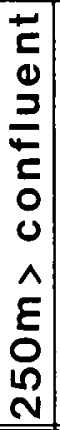 & $\begin{array}{l}m \\
\dot{N}\end{array}$ & $\stackrel{\Omega}{r}$ & $\stackrel{10}{N}$ & $\underline{0}$ & $\begin{array}{l}\bar{O} \\
\boldsymbol{N} \\
\end{array}$ & $\vec{n}$ & $\begin{array}{l}\tilde{N} \\
0 \\
0 \\
- \\
0 \\
0\end{array}$ & $\begin{array}{l}\infty \\
\sim \\
= \\
\infty \\
0 \\
0\end{array}$ \\
\hline 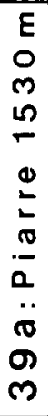 & $\begin{array}{l}E \\
E \\
0 \\
\Xi \\
E \\
E \\
0 \\
u \\
\hat{E} \\
\varepsilon \\
0 \\
0\end{array}$ & ما & 几 & $\stackrel{\sigma}{\sim}$ & 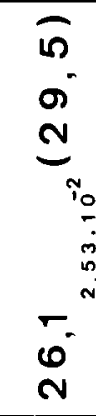 & $\begin{array}{l}\widetilde{a} \\
0 \\
\mathbb{v}\end{array}$ & $\begin{array}{l}\widetilde{F} \\
=\end{array}$ & $\begin{array}{l}\overline{0} \\
\pm \\
\dot{J}\end{array}$ & $\begin{array}{l}3 \\
\text { de } \\
\text { V }\end{array}$ \\
\hline $\begin{array}{c}E \\
E \\
0 \\
m \\
\text { in } \\
\tilde{0} \\
0 \\
\frac{1}{0} \\
0 \\
3 \\
\sigma \\
0 \\
0 \\
0 \\
\ddot{0} \\
\infty \\
\infty\end{array}$ & 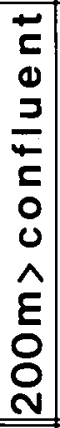 & $\begin{array}{l}n \\
1 \\
8\end{array}$ & $\stackrel{\nabla}{ }$ & $\infty$ & $\begin{array}{l}\bar{R} \\
\text { no } \\
0 \\
0 \\
0 \\
0 \\
0 \\
0 \\
0 \\
0\end{array}$ & $\begin{array}{l}\widehat{10} \\
N\end{array}$ & 6) & $\begin{array}{l}\widetilde{N} \\
=\end{array}$ & $\begin{array}{l}20 \\
\bar{V}\end{array}$ \\
\hline 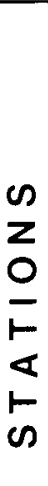 & $\begin{array}{l}z \\
0 \\
\\
\leftarrow \\
n \\
= \\
\square \\
0 \\
0 \\
J\end{array}$ & 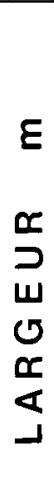 & 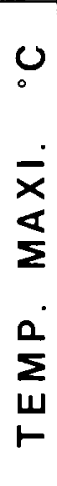 & $\begin{array}{l}w \\
\frac{1}{Z} \\
\mathbf{a}\end{array}$ & 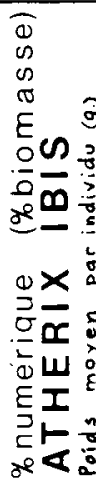 & 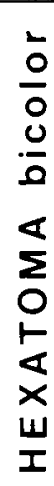 & $\begin{array}{l}0 \\
0 \\
\alpha \\
5 \\
0 \\
z \\
\alpha \\
0 \\
\frac{0}{0}\end{array}$ & $\begin{array}{l}0 \\
0 \\
0 \\
4 \\
= \\
= \\
1 \\
0 \\
0 \\
4 \\
\frac{1}{I} \\
\alpha\end{array}$ & 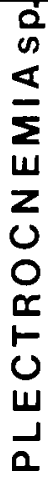 \\
\hline
\end{tabular}


Exemple 2.

Le tableau 4 met en évidence la disparition de l'Oligochète Lumbricidae Eiseniella tetraedra — dont le diamètre est du même ordre que celui des larves d'A. ibis âgées - après une cascade d'environ $130 \mathrm{~m}$ de dénivellation. $E$. tetraedra vit en particulier dans les débris de racines et de feuilles de Graminées retenus entre les pierres du fond. Ces débris ne sont pas en quantité moindre à la station inférieure (st. 2 a) mais ils y sont sans doute soumis à des contraintes mécaniques (écrasement, vibrations) plus irrégulières qu'en amont ou différentes. On note que des Hirudinées de grosse taille, aptes à se fixer solidement sur les faces supérieure ou latérales des pierres, colonisent cette station.

\section{Exemple 3.}

Le tableau 5 montre sur un autre torrent, dans des conditions assez comparables à celles du précédent cours d'eau, simultanément :

- chez les groupes dépourvus de puissants organes de fixation et pénétrant entre les pierres du fond, d'abord une disparition des formes de grosse taille (Lumbricidae), ensuite un maintien des espèces de diamètre inférieur, de l'ordre de 0,2 à $1 \mathrm{~mm}$ (Dicranota, divers Oligochètes).

- une prolifération des espèces susceptibles de se fixer activement à la surface des pierres et beaucoup moins directement tributaires de la taille des interstices (Rhyacophila).

N. B. - Exemples 2 et 3 : les prélèvements ont été effectués, en amont des cascades et dans la zone inférieure de celles-ci, dans des conditions (pierres, vitesse de courant, profondeur, éloignement des rives) aussi voisines que possible. $E$. tetraedra peut en effet subsister en été dans les graviers du bord, en courant lent (milieu très instable) ou être commun dans les touffes de Graminées et de Bryophytes bordantes immergées.

\section{4) Vitesse du courant et nature du fond.}

La nécessité pour les Athericidae d'un substrat stable est aussi un facteur limitant de la vitesse du courant. D'une façon générale, par rapport à $I$. marginata, A. ibis se trouve sur des fonds de pierres plus grosses donc par conséquent plus stables qui lui permettent de se maintenir à des viteses de courant supérieures. Quand les deux espèces cohabitent, 1 . marginata vit en général plus près des berges, dans des zones à courant plus lent.

En voici quelques exemples (mesures au micromoulinet $\mathrm{Ott}$ ) :

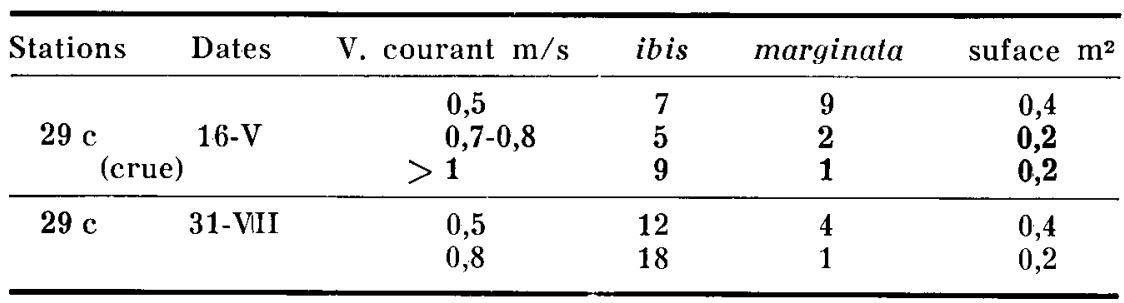




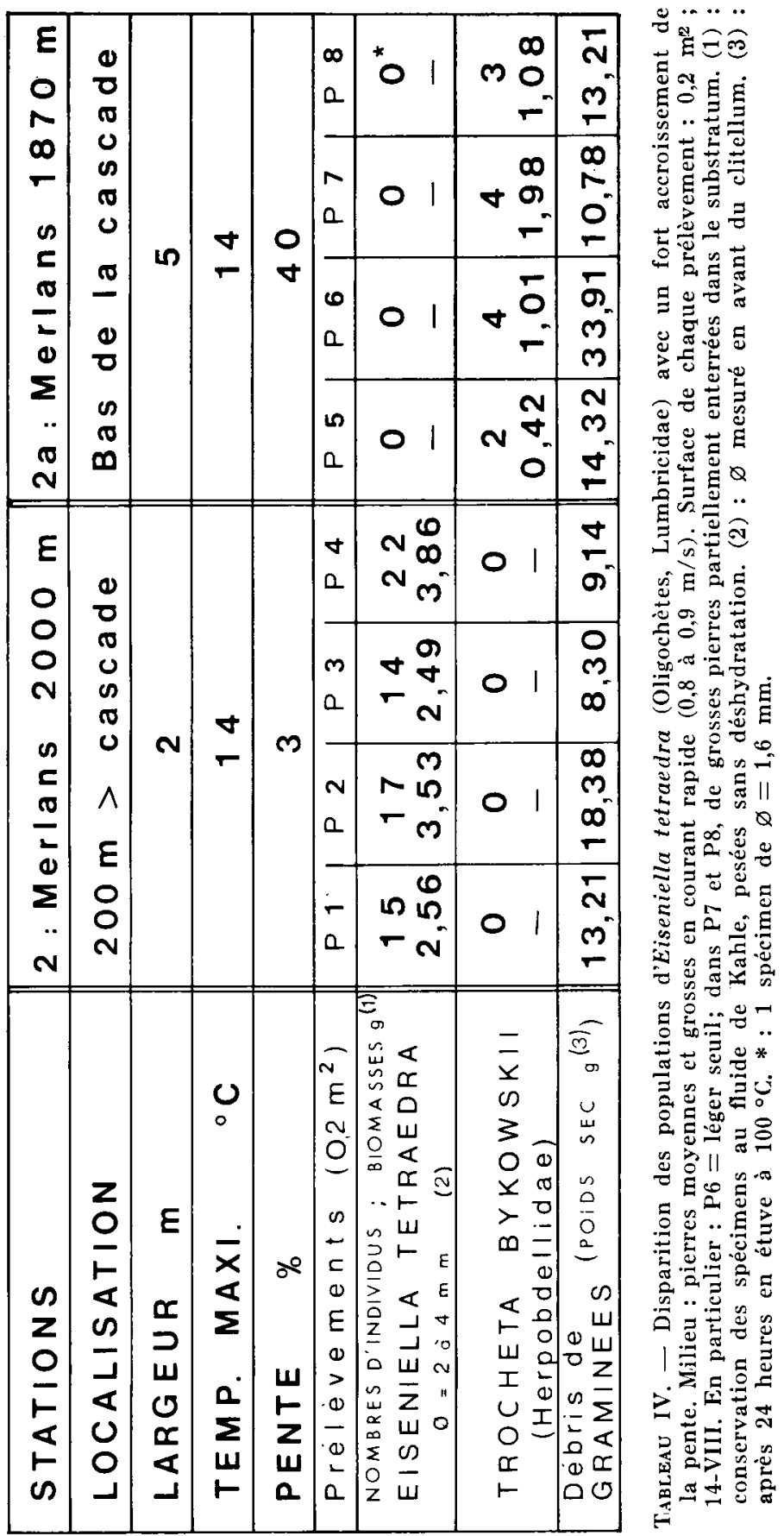




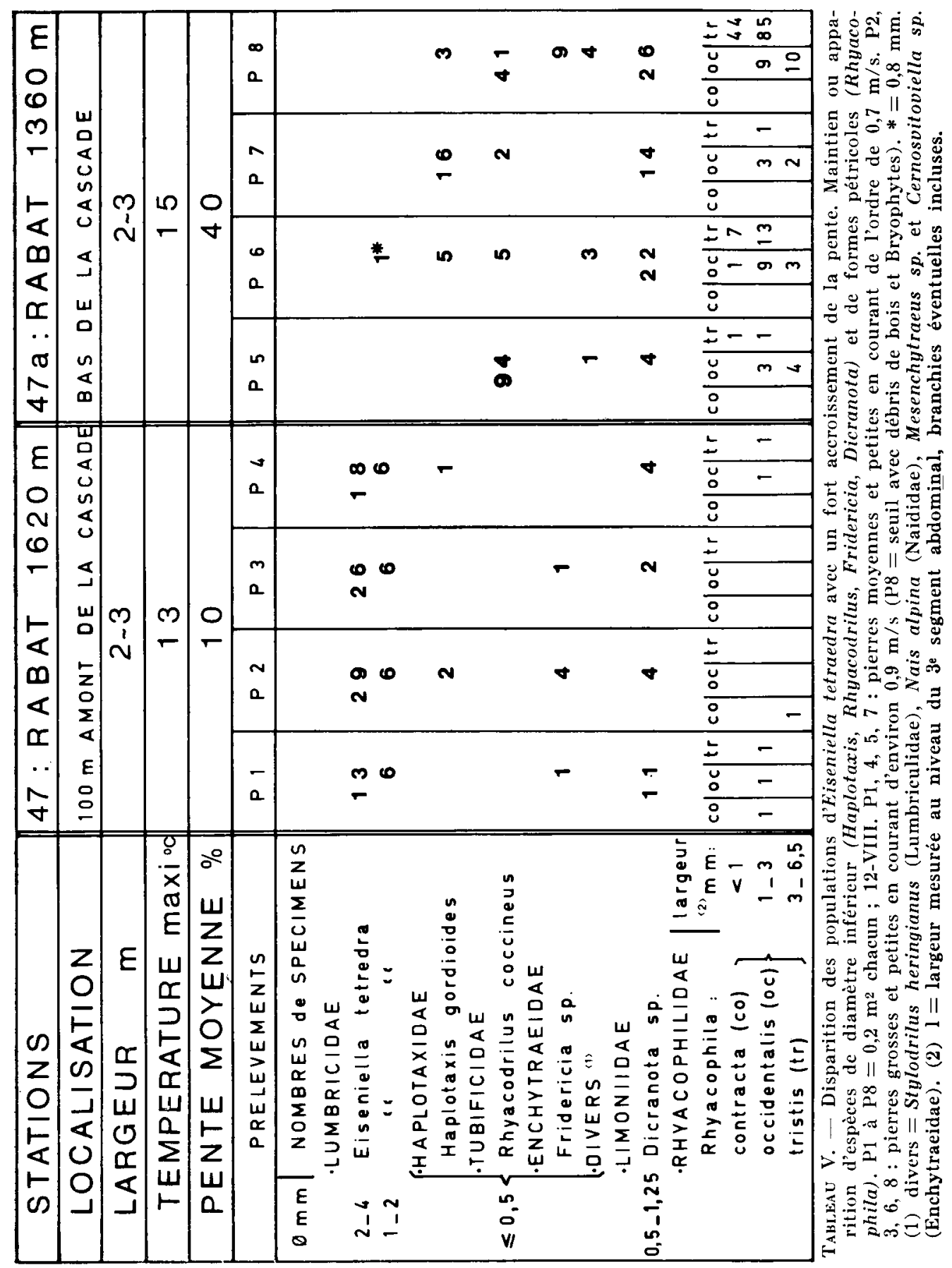




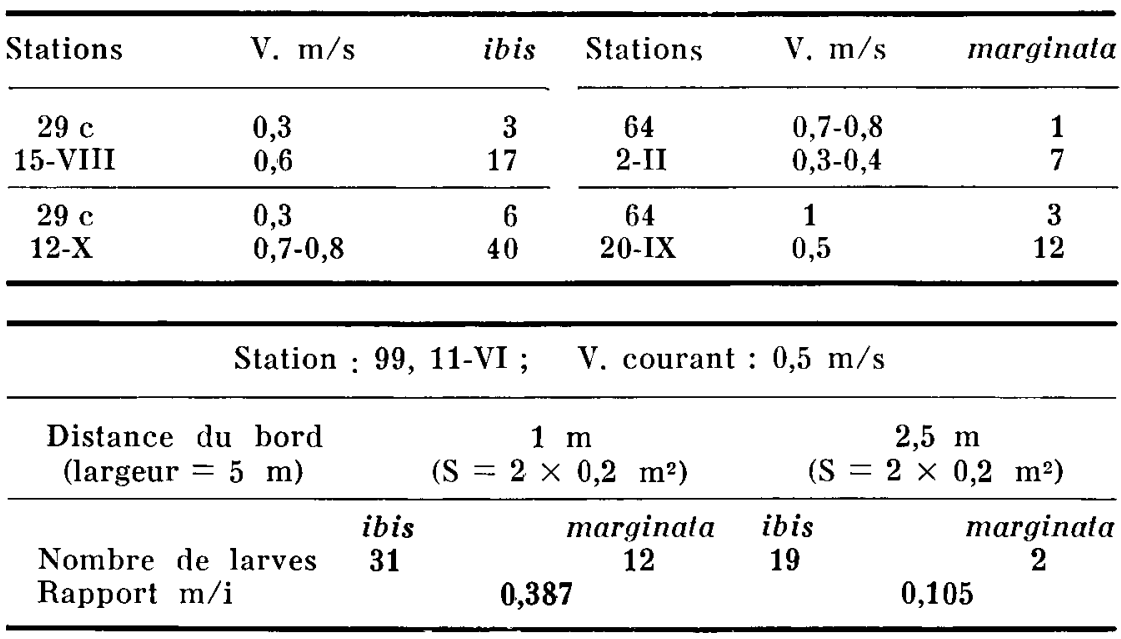

En montagne, les vitesses de courant les plus favorables, dans des conditions moyennes (par exemple de 700 à $1500 \mathrm{~m}$, pente de l'ordre de $10 \%$ ) paraissent, en dehors des crues, être voisines de $0,5 \pm 0,1 \mathrm{~m} / \mathrm{s}$ pour $I$. marginata et de 0,6 à $0,7 \pm 0,1 \mathrm{~m} / \mathrm{s}$ pour A. ibis. En plaine, I. marginata peut même se rencontrer dans des ruisseaux lents, étroits, assez envasés où elle colonise pierres et graviers. Dans les mêmes cours d'eau, ainsi que dans les grandes rivières, les fonds de sable en courant lent et parfois même temporairement nul, sont habités par $A$. crassipes qui, contrairement aux deux autres espèces, est surtout psammophile. La larve, déposée à la surface d'un sédiment immergé, s'enfonce rapidement (photos 3-4) jusqu'aux prolongements postérieurs non compris. Elle se nourrit en particulier de chironomides (Thomas $1975 \mathrm{~b}$ ) et peut constituer un élément prédateur important dans les bancs de sable bordant les cours d'eau et dans les sédiments des retenues (lacs de barrage par exemple). Elle colonise volontiers aussi les fonds pierreux avoisinants, mais en courant lent ou modéré, rarcment supérieur à $0,5 \mathrm{~m} / \mathrm{s}$; encore y est-elle toujours relativement peu abondante (tableau 1 ).

L'estimation des densités de populations d'A. crassipes dans un cours d'eau exige donc, dans la majorité des cas, d'autres techniques de prélèvement que le filet troubleau (benne Ekman, carottiers).

Exemple de station fortement peuplée par cette espèce : le Volp à $290 \mathrm{~m}$ (21-IV) :

a) Prédateurs stricts ou occasionnels dénombrés sur $625 \mathrm{~cm}^{2}$ de sable bordant immergé à la limite de battement de l'eau : A. crassipes : 24 (soit approximativement 200 fois plus que dans les pierres en courant, $20 \mathrm{~m}$ plus en aval) ; Eloophila: 1 ; Gammarides : 4. 
b) Caractéristiques du sédiment (9-VI) :

- teneur en eau : $27,24 \%$ du poids sec $\left(24\right.$ h à $\left.90^{\circ} \mathrm{C}\right)$,

- teneur en matière organique : $1,50 \%$ du poids sec $\left(2 \mathrm{~h}\right.$ à $\left.550^{\circ} \mathrm{C}\right)$,

- granulométrie : classe modale : 0,80 à $0,50 \mathrm{~mm}(23,66 \%) ; 84,85 \%$ compris entre 1,25 et $0,125 \mathrm{~mm}$; coefficient de classement d'Otto et Inman : 1,32, donc un sédiment pauvrement classé.

\section{5) Bryophytes aquatiques.}

Les larves d'A. ibis et d'I. marginata peuvent, à tous les stades, vivre dans les Bryophytes aquatiques des cours d'eau. Hubault (1927) considère même $A$. ibis et Atherix sp. (probablement $I$. marginata) comme muscicoles dans les Vosges et les Alpes.

Dans le présent travail, cet aspect n'a pas été étudié de façon approfondie, le milieu des pierres en courant paraissant, dans les Pyrénées, sinon peut être le plus riche, du moins le plus fréquemment peuplé en Athericidae. Les proportions relatives des populations muscicoles et pétricoles d'un même cours d'eau sont difficiles à déterminer, en particulier à cause de migrations possibles d'un milieu à l'autre au cours du cycle de développement. D'autre part, il est vraisemblable qu'une importance quantitative et une diversité des Bryophytes supéricures dans les ruisseaux et torrents alpins $\mathrm{y}$ entraîne un plus fort pourcentage relatif de larves muscicoles que dans les Pyrénées. Des pentes plus fortes ainsi que la possibilité pour ces larves de se développer dans un milieu légèrement plus chaud que l'eau du cours en été (mousses émergées dans la zone de battement) ne peuvent qu'accroître ce pourcentage relatif.

Quant à savoir si une espèce est plus franchement muscicole que l'autre à l'état larvaire, les résultats obtenus sur deux cours d'eau sont contradictoires : I. marginata demeure à peu près exclusivement dans les mousses (plus de $95 \%$ des individus à surface égale, le 16-IV) dans le Laudot (99) où $A$. ibis est de loin le plus abondant. A. ibis est en revanche en grande partie muscicole dans le $r$. de Sarranct à $710 \mathrm{~m}$ (13 a). En réalité, la vitesse du courant et la morphologie des Bryophytes ainsi que leur situation précise dans le lit jouent certainement un rôle important : lit régulier et vitesse plus faible dans le Laudol, mousses sur la roche en place et de gros blocs en courant rapide dans le $r$. de Sarranet.

\section{IV. - LA PONTE}

La ponte des Athericidae se fait sur un support émergé, situé directement au-dessus de l'eau. Après éclosion, les larves tombent dans le cours d'eau. 
- A. IBIS.

Si le mode de ponte colonial de cette espèce est connu depuis fort longtemps, la contribution la plus importante sur ce sujet a été celle de Nagatomi (1962). A. ibis dépose sa ponte dans un endroit abrité, en général sur un support rigide, volontiers réutilisé chaque année: tabliers de ponts, tronçons de gros tuyaux sous les chemins et en particulier anfractuosités de rochers (Nagatomi t. c., F. Vaillant comm. verb.).

L'expulsion des œufs se fait par une contraction intense des segments abdominaux qui demeurent télescopés après la mort de la $\&$ tandis que la masse d'œufs reste rattachée à l'extrémité de l'abdomen (Osmera et Spitzer 1969, photo 1). Dans mes élevages, les $q$ - en petit nombre, il est vrai -, fécondées ou non, ont toujours pondu isolément. Le processus de la ponte coloniale paraît donc tout à fait facultatif, ce qui explique la rareté des essaims dans la nature, compte tenu de la fréquente difficulté de leur trouver un abri, par exemple quand les ruisseaux coulent dans une prairie rase au-dessus de $2000 \mathrm{~m}$. Ce point est important, car l'extension de cette espèce en altitude n'est donc limitée ni par des berges rocheuses, ni par l'absence de végétation arbustive bordante. Les pontes que j'ai pu obtenir dans les bacs d'élevage avaient été déposées sur des brins de Bryophytes relativement desséchés ou sur de fines grilles plastifiées, dans les deux cas, à quelques centimètres au-dessus de l'eau.

Il est évident que l'efficacité d'une ponte collective demeure assez soumise au «tout ou rien»: les risques de destruction massive (promeneurs, pêcheurs) sont très accrus et Muttkowski (1929) note la présence de plusieurs prédateurs dans les essaims. A l'inverse, sous des conditions de développement favorables, ces essaims peuvent être à l'origine de pullulations fantastiques sans doute très temporaires — des larves pour des prédateurs torrenticoles.

\section{- I. MARginata.}

La ponte d'I. marginata est déposée à la face inférieure des feuilles, sur les basses branches des arbres bordant les cours d'eau. Ces arbries sont toujours à feuilles caduques. Je n'ai trouvé aucune ponte sur des Conifères, mais par contre à plusieurs reprises sur les essences suivantes: Acer pseudo-platanus (Acérinées), Fraxinus excelsior (Oléinées), Fagus silvatica et Corylus avellana (Cupulifères), Alnus glutinosa (Bétulinées). La face inférieure des feuilles de Corylus avellana, le noisetier, légèrement pubescente, est volontiers choisie dans le Sud de la France. La hauteur audessus de l'eau varie en général de 0,5 à $2 \mathrm{~m}$. Les pontes sont 
fréquemment éloignées le plus possible des rives, les cours d'eau étant alors à l'étiage (la période la plus favorable, dépendant peu de l'altitude semble-t-il, est la seconde quinzaine du mois de juillet).
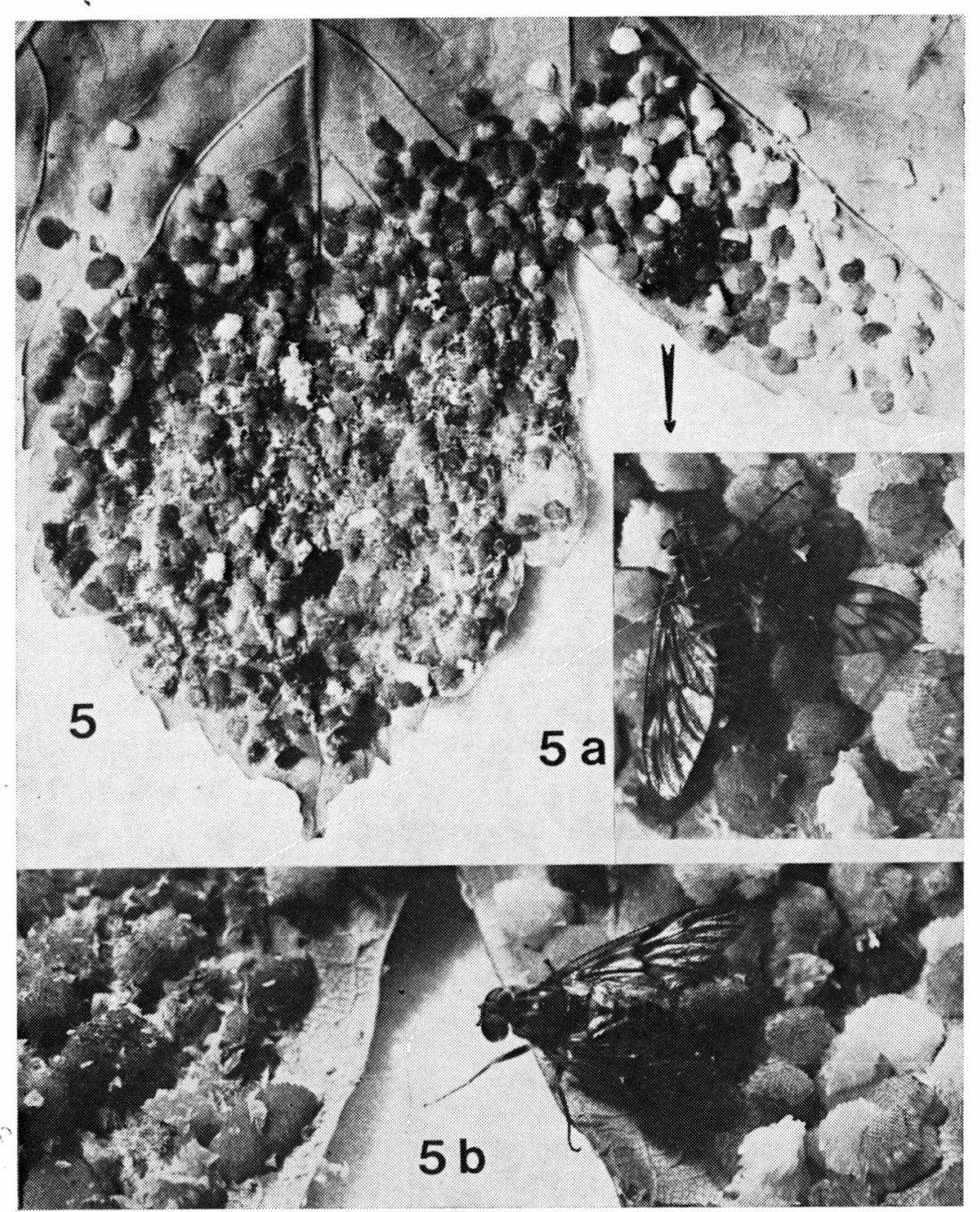

Pнотоs 5 à 5 b. - Ponte collective d'I. marginata sous une feuille de Sycomore (Acer pseudo-platanus). Noter les éclosions (5: lobe central, $5 \mathrm{~b}$ : à gauche) et la désagrégation partielle des pontes non encore écloses par les. larves néonates demeurées sur place par suite du retournement de la feuille. Présence d'une o tardive d'A. ibis déposant ses œufs parmi ceux d'I. marginata (5 a et b) puis morte in situ (5). (F. Vaillant leg.). 


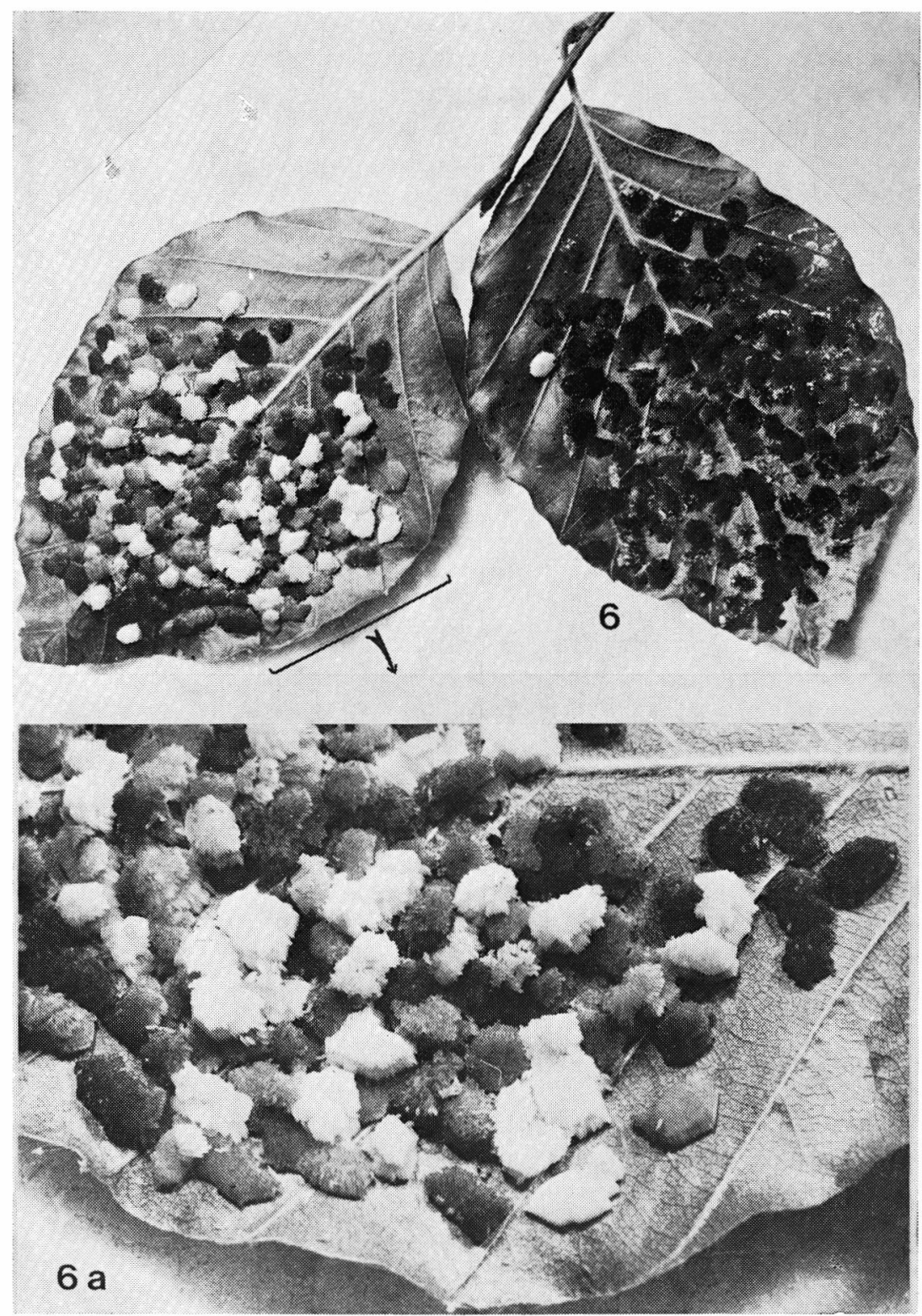

Pнотоs 6 et 6 a. - Ovipositions collectives d'I. marginata sous deux feuilles contiguës de Fagus silvatica. Noter : 1) les pontes déposées indifféremment sur un ou deux rangs ; 2) la différence de couleur entre les pontes récentes, presque blanches et les pontes en fin d'incubation, très foncées (les premières larves sortent des œufs : 6 a en haut à droite). 
Dans une zone déterminée, les pontes sont souvent situées sur une seule feuille ou un petit nombre de feuilles très rapprochées. La tendance à une oviposition collective est nette bien que facultative. Il a ainsi pu être dénombré jusqu'à plus de 160 pontes sous la même feuille de Hêtre (surface : $31 \mathrm{~cm}^{2}$ ) et plus de 400 sous une feuille de Sycomore (photos 5 et 6 ). Le nombre d'œufs portés par cette dernière peut être estimé entre 100000 et 150000 . Cette ponte coloniale (F. Vaillant leg.) provient du Cernon (Isère) à $830 \mathrm{~m}$ (température maximale pratique : $13^{\circ} \mathrm{C}$, environnement d'arbres à feuilles caduques dense). Le 31 juillet 1975 vers $16 \mathrm{~h}$ il était aisé de constater que les pontes attiraient encore plusieurs femelles d'I. marginata et même, curieusement, une $q$ tardive d'A. ibis (photo 5). Le comportement "subsocial» (Stuckenberg) d'I. marginata est donc tout à fait classique dans la lignée des Athericidae.

Dans chaque ponte, les œufs, parallèles, sont disposés à peu près perpendiculairement à la surface de la feuille sur deux rangs, parfois sur un seul, rarement sur trois. L'analogie avec beaucoup de Tabanides est donc très grande. Des comptages, effectués sur 10 pontes nettement isolées des autres, font apparaître un nombre moyen de 301 œufs, pour un minimum de 188, un maximum de 456 et un écart type de 84 . Les pontes disposées sur deux rangs ne renferment pas davantage d'œufs que les autres et l'importance du rang apical - quand il existe - par rapport au total est aussi très variable : 14 à $37 \%$ sur 6 pontes seulement.

Les oufs mesurent environ $1150 \mu$ de longueur et 220 de diamètre maximum. Clairs lors de l'oviposition, ils foncent au cours du développement (pontes à divers états de maturité sur la photo 6), en particulier dans la région apicale où se produit, lors de l'éclosion, une déhiscence longitudinale et seulement dorsale sur le quart ou le tiers de la longueur totale. Les éclosions des oufs d'une même ponte sont le plus souvent massives et se produisent de proche en proche; mais elles peuvent aussi être étalées sur 2 ou 3 jours. La durée de l'incubation - mesurée sur une ponte à l'origine de 3 larvules seulement - est de 15 jours à une température constante de $18{ }^{\circ} \mathrm{C}$ et une faible hygrométrie. Les masses d'œufs sont souvent largement superposées, ce qui favorise à la fois la mort des jeunes larves des pontes sous-jacentes, plus anciennes donc écloses plus tôt, et la destruction des pontes superficielles avant leur éclosion. A plusieurs reprises, j'ai pu constater que, de la face inférieure des feuilles, les larves néonales tombent immédiatement dans l'eau où elles sont tout à fait capables de survivre. Ces observations ne vont pas dans le sens de celles de Pleskot (citée par Oldroyd 1964) selon laquelle les jeunes larves se maintiendraient sur les feuilles presque jus- 
qu'au second stade avant de rejoindre le ruisseau. En plein été, la déshydratation cause la mort des larvules en quelques heures tout au plus. En outre, leur mortalité est rapidement considérable lorsqu'elles sont maintenues en grand nombre sur une surface de quelques $\mathbf{c m}^{2}$ : elles se débattent violemment, malgré une tendance à rester groupées en amas denses.

Bien que la ponte puisse aussi avoir lieu sur la face inférieure de Graminées de grande taille en surplomb de l'eau (une seule observation : $96 \mathrm{c}$, en fin de période de vol, 15-VIII), le mode d'oviposition apparaît comme un important facteur limitant de la répartition d'I. marginata en altitude, dans le sud de la France. Dans le tableau 1, les exceptionnelles citations de cette espèce audessus de la limite des arbres à feuilles caduques (2 sur 91) pourraient être à attribuer à des migrations ascendantes de rares larves (classe d'abondance : $P$ ) en l'absence de cycle de développement complet $(38$ a, 75$)$.

\section{- A. crassipes.}

La ponte d'A. crassipes n'a pas été observée. Elle n'exige vraisemblablement pas de manière absolue, la présence de végétation arbustive bordante. Nagatomi (1962) a signalé la ponte des deux espèces japonaises d'Atrichops sous une fougère et plusieurs arbres et arbustes.

\section{V. - LA NYMPHOSE}

Les nymphes des Athericidae sont totalement ignorées des hydrobiologistes. La recherche des exuvies nymphales constitue cependant le moyen le plus commode à la fin de l'été pour déceler la présence d'l. marginata dans un petit cours d'eau.

La nymphose des trois espèces est aérienne. Elle est compromise si le milieu où elle se déroule est envahi par l'eau, même temporairement. La larve âgée migre donc en direction des berges où la nymphose a lieu bien au-dessus du niveau normal de l'eau, pour éviter les conséquences de crues éventuelles.

\section{- I. Marginata.}

Chez cette espèce, la nymphose se fait dans les Bryophytes bordant le cours et peut-être aussi dans les touffes de Graminées humides. La distance parcourue par les larves à partir du niveau de l'eau est aisément mesurable sur les parois rocheuses verticales ou fortement inclinées car les exuvies nymphales émergent des mousses jusqu'au $2^{\mathrm{e}}$ ou $3^{\mathrm{e}}$ segment abdominal et sont très appa- 
rentes. Cette distance est presque toujours comprise entre 0,5 et $1,5 \mathrm{~m}$, quelques nymphes atteignant $2 \mathrm{~m}$ de hauteur au-dessus de l'eau (43).

En élevage, la mortalité des nymphes dans d'autres milieux que les Bryophytes s'est révélée élevée. Par suite, indirectement, la dépendance d'I. marginata vis-à-vis des couverts de feuillus denses est encore accrue, ce qui explique pourquoi les deux plus fortes classes d'abondance ne sont pratiquement pas atteintes dans les ruisseaux de plus de $2 \mathrm{~m}$ de largeur (tableau 1 et fig. $1 \mathrm{~d}$ ). En effet, avec l'espacement croissant des rives, la voûte constituée par le couvert d'arbres devient de plus en plus clairsemée : l'insolation augmente alors dans de fortes proportions, ce qui entraîne la régression des Bryophytes.

\section{- A. IBIS.}

Au printemps, il est aisé de constater dans les élevages que la larve âgée se recroqueville dans les graviers bordants humides et $\mathbf{y}$ demeure immobile, ou à peu près, pendant plusieurs semaines. Nagatomi (1962) a appelé cet état «prepupa». Ce terme est approprié, car la larve se montre encore capable de mouvements, par exemple lorsqu'elle est dérangée. La durée de cet état, très variable selon Nagatomi, a été en général considérable dans mes élevages (jusqu'à 2 mois). Je pense qu'elle doit être directement en rapport avec la teneur en eau des graviers.

Sur le terrain, j'ai pu observer des prénymphes hors de l'cau en avril, dans les graviers bordants (99). Granulométrie : 45,58\% au-dessus de $5 \mathrm{~mm}$ et $72,25 \%$ au-dessus de $1,25 \mathrm{~mm}$.

La transformation en nymphe proprement dite n'a lieu qu'ensuite; elle est alors apparente en peu de temps au niveau de la région antérieure (environ $24 \mathrm{~h}$ ).

La nymphose peut se dérouler, comme chez 1 . marginata, dans les Bryophytes bordants, mais ce milieu est tout à fait facultatif.

\section{Exemple :}

Des larves ont été placées en mars entre les pierres d'un bac au débit réduit à un filet d'eau. Au préalable, ces pierres, alignées longitudinalement dans la partie centrale du bac, avaient été déposées sur la zone de contact entre d'une part les Bryophytes recouvrant l'une des faces en $V$ sur 2 à $3 \mathrm{~cm}$ d'épaisseur et d'autre part les graviers recouvrant l'autre face sur 1 à $2 \mathrm{~cm}$. 6 nymphes et 6 émergences ont été obtenues: 3 dans les Bryophytes, 3 dans les graviers. Granulométrie de ces derniers : $38,61 \%$ au-dessus de $5 \mathrm{~mm}, 46,77 \%$ entre 5 et $2 \mathrm{~mm}$, $14,62 \%$ entre 2 et $0,5 \mathrm{~mm}$. Dans les bacs dépourvus de Bryophytes et dotés uniquement d'un fond de graviers, les émergences sont obtenues très facilement avec une mortalité peu importante. 
Sur le terrain, j'ai pu récolter des exuvies nymphales dans les graviers du bord, très près de l'eau (66 a).

On peut donc dire que la nymphose d'A. ibis est plus indépendante de la nature des biotopes que celle d'I. marginata. Elle est aussi mieux adaptée aux hautes altitudes où les Bryophytes sont moins fréquents et moins abondants.

\section{一 A. CRassipes.}

Au cours des élevages, 11 nymphes et 11 imagos de cette espèce ont été obtenues, toutes dans le sable, dont une faible épaisseur est suffisante (moins de $5 \mathrm{~mm}$ ). Ce sable, sur plan incliné, était presque sec ou même désagrégé. La durée de la nymphose, mesurée dans ces conditions pour 2 \% a été d'un peu plus de 10 jours et de près de 12 jours à $19^{\circ} \mathrm{C}$.

A la station $96 \mathrm{c}$ (milieu décrit au $\S \mathrm{III}, 4$ ), les migrations ont lieu au plus tard au début juin : un prélèvement effectué le 9-VI sur $3000 \mathrm{~cm}^{2}$ n'a fourni que 2 larves. Par contre, l'année précédente, exactement au même endroit, toutes les larves recueillies le 5-VI (9) se trouvaient dans le sable émergé en pente douce, entre $60 \mathrm{~cm}$ et $1 \mathrm{~m}$ du bord de l'eau. Elles étaient encore assez actives et paraissaient plutôt être des individus en cours de migration que des prénymphes.

\section{Remarque.}

La nymphe d'I. marginata possède sur l'abdomen des prolongements ventraux beaucoup plus longs que ceux des deux autres espèces (voir Thomas $1974 \mathrm{~b}$, fig. 13) et souvent brisés lorsque l'exuvie est retirée des Bryophytes. Par rapport aux fortes épines correspondantes d'A. ibis et d'A. crassipes, ces prolongements constitucnt une adaptation à la fixation dans un milieu plus aéré, beaucoup moins dense que les sables ou les graviers.

\section{VI. - CONCLUSION}

Du point de vue hydrobiologique, les Athericidae ont été jusqu'ici un groupe à peu près oublié. La présente étude montre que deux espèces de cette famille (Atherix ibis et Ibisia marginata) sont très répandues, en particulier en moyenne montagne, et que les densités des populations sur fonds de pierres sont dans l'ensemble bien loin d'être négligeables pour des prédateurs.

Sur 237 stations, 132 sont habitées par au moins une espèce d'Athericidae (I. marginata : 86 stations ; A. ibis : 67; A. crassi- 
pes : 25). Ce nombre est à rapprocher des 127 stations colonisées par les Limoniidae des genres torrenticoles les plus répandus (Dicranota : 104 et Hexatoma : 78). Les Rhagionidae (Chrysopilus : 31 ) demeurent beaucoup plus localisés.

\section{TRAVAOX CITES}

Badcock (R. M.). 1949. - Studies in stream life in tributaries of the Welsh Dee. J. Anim. Ecol., 18 : 193-208.

BERTHÉlemy (C.). 1966. - Recherches écologiques et biogéographiques sur les Plécoptères et Coléoptères d'eau courante (Hydraena et Elminthidae) des Pyrénées. Annls Limnol., 2 (2) : 227-458.

Berthélemy (C.) et Laur (C.). 1975. - Plécoptères et Coléoptères aquatiques du Lot (Massif Central français). Annls Limnol., 11 (3) : 263-285.

Brindle (A.). 1961. - Taxonomic Notes on the Larvae of British Diptera. 4. The genus Atherix Meigen (Rhagionidae). Entomologist, $94: 218-220$.

Décamps (H.). 1967. - Écologie des Trichoptères de la Vallée d'Aure (Hautes-Pyrénées). Annls Limnol., 3 (3) : 399-577.

DitTmar (H.). 1955. - Ein Sauerlandbach. Untersuchungen an einem Wiesen-Mittelgebirgsbach. Arch. Hydrobiol., 50 : 305-552.

Hubault (E.). 1927. - Contribution à l'étude des Invertébrés torrenticoles. Bull. biol. Fr. Belg., suppl. IX : 389 p. +10 pl.

Jones (J. R. E.). 1949. - An ccological study of the River Rheidol, North Cardiganshire, Wales. J. Anim. Ecol., $18: 67-88$.

Lavandier (P.). 1974. - Écologie d'un torrent pyrénéen de haute montagne. I. Caractéristiques physiques. Annls Limnol., 10 (2) : 179219.

Mutrkowski (R. A.). 1929. - The ecology of trout streams in Yellowstone National Park. Roosevelt Wild Life Ann., 2 (2) : 151-240.

Nagatomi (A.). 1958. - Studies in the aquatic Snipe-flies of Japan. Part I. Descriptions of the adult (Diptera, Rhagionidae). Mushi, 32 (5) : 47-67.

Nigitomi (A.) 1960. - Studies in the aquatic Snipe-flies of Japan. Part II. Descriptions of the eggs (Diptera, Rhagionidae). Mushi, $33(1): 1-3$.

Nagatom (A.). 1961 a. - Studies in the aquatic Snipe-flies of Japan. Part III. Descriptions of the larvae (Diptera, Rhagionidae). Mushi, 35. (3) : 11-27.

Nagatomi (A.). $1961 \mathrm{~b}$. - Studies in the aquatic Snipe-fllies of Japan. Part IV. Descriptions of the pupae (Diptera, Rhagionidae). Mushi, 35 (4) : 29-38.

Nagatom (A.). 1962. - Studies in the aquatic Snipe-flies of Japan. Part V. Bilogical notes. Mushi, 36 (10) : 103-149.

Neveu (A.). - Ecologie des larves d'Athericidae (Diptera, Brachycera) dans un ruisseau des Pyrénées-Atlantiques. I. Structure et dynamique des populations. Annls Hydrobiol. (Sous presse.)

Nietzke (G.). 1938. - Die Kossau. Hydrobiologische-faunistische Untersuchungen an schleswig-holsteinischen Fliessgewässern. Arch. Hydrobiol., 32 : 1-74. 
Oldroyd (H.). 1964. - The Natural History of Flies. Weidenfeld et Nicolson. Londres. 324 p. +32 pl.

Osmera (S.) et Spitzer (K.). 1969. - Cíhalka Atherix ibis (F.) (Diptera, Rhagionidae) $v$ jižních čechách. Sbornik Jihočeského muzea $v$ Ceských Budějovicich Př́irodni vědy. IX : 34-38 +3 pl.

Pomeisl (E.). 1953 a. - Der Mauerbach. Wett. Leben, 2 : 103-121.

PoMeisi, (E.). 1953 b. - Studien an Dipterenlarven des Mauerbaches. Wett. Leben, 2 : 165-176.

SÉGuy (E.). 1926. - Diptères Brachycères. In : Faune de France (13). Paris, Lechevalier, 308 p.

Sow (R.). 1965. - Ecological characteristics of the bottom fauna of the Wielka Puszcza Stream. Acta hydrobiol., 7, suppl. 1 : 61-92.

Stuckenberg (B. R.). 1974. - A new genus and two new species of Athericidae (Diptera) in Baltic Amber. Ann. Natal Mus., 22 (1): 275-288.

Thomas (A. G. B.). 1974 a. - Diptères torrenticoles peu connus. I. Les Athericidae (larves et imagos) du Sud de la France (Brachycera, Orthorrhapha). Annls Limnol., 10 (1) : 55-84.

Thomas (A. G. B.). 1974 b. - Diptères torrenticoles peu connus. II. Les Athericidae (nymphes) du Sud de la France (Brachycera, Orthorrhapha). Annls Limnol., 10 (2) : 121-130.

Thomas (A. G. B.). 1975 a. - Ephéméroptères du Sud-Ouest de la France. I. Migrations d'imagos à haute altitude. Annls Limnol., 11 (1) : 47-66.

Thomas (A. G. B.). 1975 b. - Diptères torrenticoles peu connus. III. Les Athericidae du Sud de la France (régime alimentaire des larves : aspect qualitatif) (Brachycera, Orthorrhapha). Annls Limnol., 11 (2) : 169-188.

VAn̆haRA (J.). 1975. - Larval development of Atherix marginata (Diptera, Athericidae) under natural conditions. Acta ent. bohemoslov., 72 (2) : 92-98.

Zaćwilichowska (K.). 1970. - Diptera larvae in the River San and some of its tributaries. Acta Hydrobiol., 12 (2-3) : 197-208. 